\title{
LUT
}

University

\section{Analysis of Electromagnetic Excitations in an Integrated Centrifugal Pump and Permanent Magnet Synchronous Motor}

Kim Heesoo, Posa Atte, Heikkinen Janne, Nerg Janne, Sopanen Jussi

This is a Final draft

version of a publication

published by IEEE

in IEEE Transactions on Energy Conversion

DOI: $\quad 10.1109 /$ TEC.2019.2935785

Copyright of the original publication: (C) IEEE 2019

Please cite the publication as follows:

Kim, H., Posa, A., Heikkinen, J., Nerg, J., Sopanen, J. (2019). Analysis of Electromagnetic Excitations in an Integrated Centrifugal Pump and Permanent Magnet Synchronous Motor. IEEE Transactions on Energy Conversion. DOI: 10.1109/TEC.2019.2935785

(C) 2019 IEEE. Personal use of this material is permitted. Permission from IEEE must be obtained for all other uses.

This is a parallel published version of an original publication.

This version can differ from the original published article. 


\title{
Analysis of Electromagnetic Excitations in an Integrated Centrifugal Pump and Permanent Magnet Synchronous Motor
}

\author{
Heesoo Kim ${ }^{\circledR}$, Atte Posa, Janne Nerg ${ }^{\circledR}$, Senior Member, IEEE, Janne Heikkinen, \\ and Jussi T. Sopanen (1), Member, IEEE
}

\begin{abstract}
The effect of unbalanced magnetic pull (UMP) caused by air gap eccentricity on the vibration of a permanent magnet synchronous motor (PMSM) is investigated. The force model is established analytically by the Maxwell stress method. For accurate consideration of the eccentricity condition, mixed eccentricity, axial-varying eccentricity, and eccentricity caused by motor frame vibration are modeled and combined. The model of the rotorbearing system, which includes the UMP model, is developed with two different methods. In the first method, UMP is added as a linear negative spring to the rotor model, whereas in the second method, UMP is included as an external force. The rotor system of a centrifugal pump driven by an integrated PMSM is modeled using beam elements, and the two distinct modeling approaches for UMP are applied. From the results, the UMP effect on vibration and the difference between the two modeling methods are investigated. To verify the results of the analysis, experimental work is done with a pump test rig, and results of frequency spectra are obtained. Based on the analyses and experimental work, the negative stiffness effect and additional vibration excitations caused by UMP are examined.
\end{abstract}

Index Terms-Axial-varying eccentricity, Eccentricity by frame vibration, Permanent magnet synchronous motor, Mixed eccentricity, Unbalanced magnetic pull.

$\mathbf{E}$ LECTROMECHANICAL interaction in rotating electrical machines is a significant factor in the generation of nonlinear dynamic behavior of a system. In machines with a small air gap, such nonlinear dynamic behavior can be dangerous for the rotor system, and therefore, many studies have been conducted on unbalanced magnetic pull (UMP) caused by electromechanical interaction.

The topic of UMP has been addressed in numerous studies covering various factors such as asymmetry of rotor and stator, rotor eccentricity, and magnetic saturation. Ortega et al. [1]

Manuscript received July 6, 2018; revised May 28, 2019; accepted July 15, 2019. This work was supported by LUT Doctoral School Funding. Paper no. TEC-00728-2018. (Corresponding author: Heesoo Kim.)

H. Kim, J. Heikkinen, and J. T. Sopanen are with the Department of Mechanical Engineering, LUT University, 53850 Lappeenranta, Finland (e-mail: heesoo.kim@lut.fi; janne.heikkinen@lut.fi; jussi.sopanen@lut.fi).

A. Posa is with the Sulzer Pumps Finland Oy, 48600 Kotka, Finland (e-mail: atte.posa@sulzer.com).

J. Nerg is with the Department of Electrical Engineering, LUT University, 53850 Lappeenranta, Finland (e-mail: janne.nerg@lut.fi).

Color versions of one or more of the figures in this article are available online at http://ieeexplore.ieee.org.

Digital Object Identifier 10.1109/TEC.2019.2935785 conducted experiments and a finite element analysis (FEA) to study the effects of asymmetries caused by manufacturing tolerances of the stator and rotor magnets on the performance of a PMSM. Zhu et al. [2] developed a general analytical model to predict UMP in a permanent magnet brushless AC and DC machines having a diametrically asymmetric disposition of slots and phase windings. Liu et al. [3] developed an analytical model including the effect of interaction between the pole transitions and the slot openings to analyze permanent magnet motors with a slotted stator core. The rotor eccentricity, in particular, has been widely investigated as a factor contributing to the UMP. Donat [4] calculated UMP caused by air gap eccentricity based on an electromagnetic-coupled field analysis in the Ansys software. Dorrell et al. [5] considered a combination of static and dynamic eccentricities and [6] studied a method for calculating UMP in cage induction motors; the model includes magnetic saturation and axial variation with static or dynamic eccentricity. Li et al. [7] modeled axial-varying eccentricity by a superposition method and verified the model by comparing its results with 3D FEA results. Tenhunen et al. [8] investigated UMP in an induction motor when the rotor is in whirling motion by using a method based on the principle of virtual work and measured it for a test motor supported by active magnetic bearings. Guo et al. [9] obtained analytical expressions of UMP by air gap eccentricity for any pole pair number. Di et al. [10] modeled the curved dynamic eccentricity caused by a bent rotor.

The effect of UMP has been investigated by studying the dynamic behavior of a rotor system. Chen et al. [11] studied the analytical UMP calculation method considering the magnetomotive force (MMF) of the rotor and the stator in a PMSM. They discussed the stability of the steady response by using an eigenvalue analysis for the Jeffcott rotor. Xiang et al. [12] studied the stiffness characteristics and nonlinear dynamic behavior of the Jeffcott rotor system of a PMSM affected by UMP. Losak et al. [13] modeled UMP as a spring element and investigated the rotor deflection and critical speed. Xu et al. [14] examined a rotor model considering both static and dynamic eccentricity. They compared the results with a case including dynamic eccentricity only and found that vibration displacement is increased and the rotor shaft orbit is no longer centrosymmetric and is only axisymmetric in the direction of the static eccentricity. Pennacchi [15] studied a UMP model based on the actual position of the rotor not limited to circular orbits and validated the proposed 
model by measuring the dynamical behavior of a steam turbo generator.

The rotor eccentricity is an important cause of UMP and simultaneously, a result of various factors, such as manufacturing tolerances, faults, and rotor whirling motion. Therefore, accurate modeling of eccentricity is a prerequisite for a UMP study. In most previous studies, the factors causing the rotor eccentricity have been studied individually and the effect of UMP has been investigated for a Jeffcott rotor system. In actual systems, however, different eccentricity-generating factors occur simultaneously and the total eccentricity is a result of complex interactions. Therefore, in this study, a combined model with mixed eccentricity, axial-varying eccentricity, and eccentricity caused by motor frame vibration is first developed and the UMP model with combined eccentricity is then applied to a rotor-bearing model for an actual prototype electrical machine with two different approaches, and its effects are studied. The results are verified by a comparison with experimental results for a pump test rig.

\section{ECCENTRICITY MODELING}

In the conventional analysis, two special cases of whirling motion, i.e., static eccentricity and dynamic eccentricity, are typically studied as air gap eccentricity. In static eccentricity, the whirling frequency is zero and the eccentricity results from manufacturing tolerances, wear, and misalignment of bearings. In dynamic eccentricity, the whirling frequency is equal to the rotation speed of the rotor, and the center axis of whirling motion is the same as the center axis of the stator. Such eccentricity typically results from a bent shaft or unbalance mass of the rotor.

In a real system, eccentricity is a result of a combination of both static and dynamic eccentricities and not identical in the axial direction of an eccentric rotor. Moreover, eccentricity is affected by frame vibration. To demonstrate this condition, these eccentricities are modeled individually and combined.

\section{A. Mixed Eccentricity}

Under the assumption that the rotor and the stator are ideal cylinders and the stator is rigid and does not vibrate, air gap eccentricity can be defined in the form of mixed eccentricity by combining the static and dynamic eccentricities. Mixed eccentricity can be explained as a condition in which the rotor rotates with a certain whirling amplitude about the eccentric axis, which is displaced from the center axis of the stator bore, i.e., eccentricity between the stator bore and the bearing bore center is given as a static condition, and the rotor rotates about the bearing bore center with a whirling amplitude. Therefore, mixed eccentricity can be defined as a displacement vector of the rotor center with the stator bore center as the initial point. It is dependent on time and expressed as magnitude $e_{0}$ and direction angle $\theta_{0}$.

To model this condition, two reference coordinate systems are defined as in Fig. 1. The origin $O_{1}$ of the $x_{1}-y_{1}$ coordinate system is the geometric center of the stator, and the origin $\mathrm{O}_{2}$ of the $x_{2}-y_{2}$ coordinate system is the center of rotor whirling and can also be regarded as the geometric center of the bearings. $O_{\mathrm{r}}$ is the geometric center of the rotor. The $x_{2}-y_{2}$ coordinate

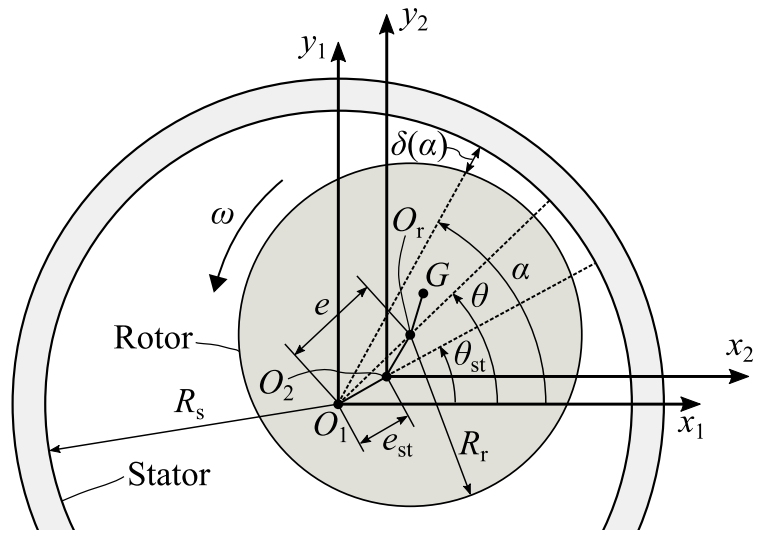

Fig. 1. Cross-sectional view of an eccentric rotor. ( $G$ : rotor mass center).

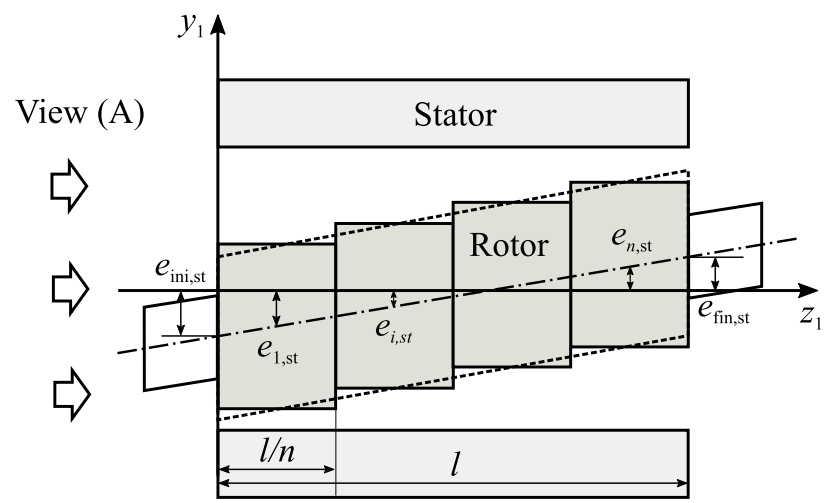

Fig. 2. Finite element model representation of an axial-varying static eccentric rotor (dotted line: real rotor condition).

system is translated parallel to the $x_{1}-y_{1}$ coordinate system as the same degree as the static eccentricity. Therefore, the rotor displacement can be defined as $x_{2}$ and $y_{2}$ coordinates of the rotor center $O_{\mathrm{r}}$, and the mixed eccentricity can be defined by combining static eccentricity and rotor displacement. Therefore, the magnitude and direction angle of mixed eccentricity are defined as

$$
\begin{aligned}
& e(t)=\sqrt{\left(e_{\mathrm{st}} \cos \theta_{\mathrm{st}}+x_{2}(t)\right)^{2}+\left(e_{\mathrm{st}} \sin \theta_{\mathrm{st}}+y_{2}(t)\right)^{2}} \\
& \theta(t)=\tan ^{-1}\left(\frac{e_{\mathrm{st}} \sin \theta_{\mathrm{st}}+y_{2}(t)}{e_{\mathrm{st}} \cos \theta_{\mathrm{st}}+x_{2}(t)}\right)
\end{aligned}
$$

where $e_{\text {st }}$ and $\theta_{\text {st }}$ are the amplitude and the direction angle of static eccentricity, respectively. The coordinates $x_{2}$ and $y_{2}$ denote the instantaneous displacements of the rotor center.

\section{B. Axial-Varying Eccentricity}

In an actual system, air gap eccentricity is not consistent with the axial direction because of manufacturing tolerances and assembly misalignment. On the other hand, for calculation of UMP, it must be assumed that the air gap is consistent with the axial direction. Because the magnitude of the UMP is proportional to the axial length of the electrical active rotor, the rotor can be modeled as divided elements having axially consistent and individual eccentricity, as in Fig. 2. To define the mixed eccentricity in this finite element model, the static 


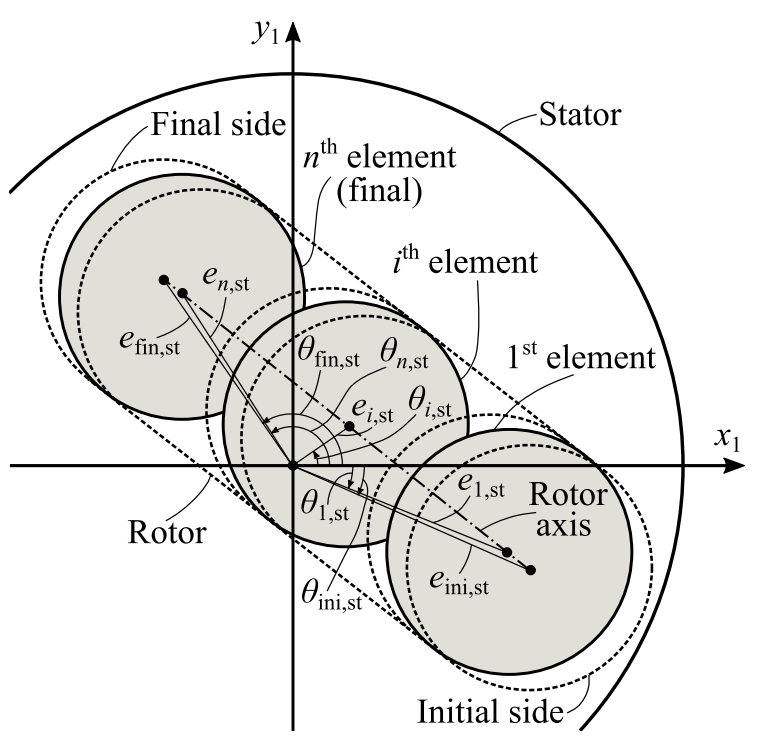

Fig. 3. View (A) of the finite element model with axial-varying static eccentricity.

eccentricities $\left(e_{i, \mathrm{st}}, \theta_{i, \mathrm{st}}\right)$ for all elements of the rotor have to be determined. If the rotor is not bent, the static eccentricity of an arbitrary intermediate rotor element can be calculated from the eccentricities of the initial and final side using the geometric relationship presented in Fig. 3. Static eccentricity for an arbitrary $i$ th element is defined as in (3) and (4).

$$
\begin{aligned}
& e_{i, \mathrm{st}}=\sqrt{x_{i, \mathrm{st}}^{2}+y_{i, \mathrm{st}}^{2}} \\
& \theta_{i, \mathrm{st}}=\tan ^{-1}\left(\frac{y_{i, \mathrm{st}}}{x_{i, \mathrm{st}}}\right), \text { when }-\frac{\pi}{2}<\theta<\frac{\pi}{2}
\end{aligned}
$$

in which

$$
\begin{aligned}
x_{i, \mathrm{st}}= & e_{\mathrm{ini}, \mathrm{st}} \cos \theta_{\mathrm{ini}, \mathrm{st}} \\
& +\frac{2 i-1}{2 n}\left(e_{\mathrm{fin}, \mathrm{st}} \cos \theta_{\mathrm{fin}, \mathrm{st}}-e_{\mathrm{ini}, \mathrm{st}} \cos \theta_{\mathrm{ini}, \mathrm{st}}\right) \\
y_{i, \mathrm{st}}= & e_{\mathrm{ini}, \mathrm{st}} \sin \theta_{\mathrm{ini}, \mathrm{st}} \\
& +\frac{2 i-1}{2 n}\left(e_{\mathrm{fin}, \mathrm{st}} \sin \theta_{\mathrm{fin}, \mathrm{st}}-e_{\mathrm{ini}, \mathrm{st}} \sin \theta_{\mathrm{ini}, \mathrm{st}}\right)
\end{aligned}
$$

where $x_{i, \mathrm{st}}$ and $y_{i, \mathrm{st}}$ are the coordinates of the center of the $i$ th rotor element with respect to the $x_{1}-y_{1}$ coordinate system. The number of divided rotor elements is $n$, and therefore, $i=1,2$, $\ldots, n$. The static eccentricity of the initial side of the rotor is given by $e_{\mathrm{ini}, \mathrm{st}}$ and $\theta_{\mathrm{ini}, \mathrm{st}}$, whereas $e_{\mathrm{fin}, \mathrm{st}}$ and $\theta_{\mathrm{fin}, \mathrm{st}}$ present the static eccentricity of the final side of the rotor. Consequently, the axial-varying eccentricity condition is modeled by using divided elements that have individual eccentricity. Moreover, this model can be easily applied to the rotor simulation model using finite elements.

\section{Eccentricity by Motor Frame Vibration}

The UMP excites both the stator and the rotor. At the same time, the vibration of the motor frame changes the air gap

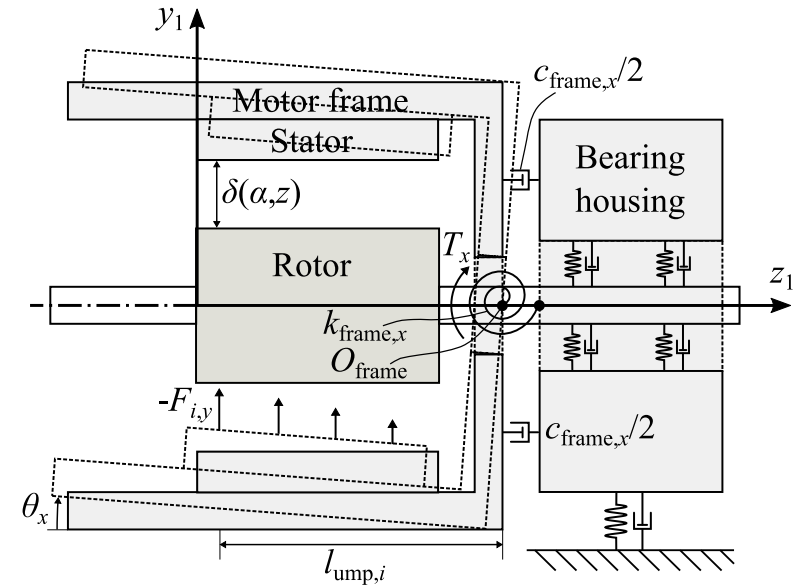

Fig. 4. Motor frame vibration model of the machine under study.

eccentricity. Therefore, it is necessary to predict the vibration behavior of the motor frame and consider its effect on the eccentricity. In this study, a simple model for the whole frame structure is developed to be easily applied to the air gap eccentricity calculation process. First, for the frame structure of the machine under study, a modal analysis was performed with ANSYS, and as a result, two frame-dominated modes were found [16]. Based on these mode shapes, it is assumed that the motor frame and the stator are rigid and considered one body, and the bearing housing is rigid and connected rigidly to the ground. This approach is taken as the focus of interest is on the effect of relative rotational vibration between the motor frame and the bearing housing. Based on these assumptions, the frame structure consisting of the motor frame and the bearing housing and excited by the UMP is modeled as a two-degree-of-freedom system as in Fig. 4. In this model, the motor frame is connected with the bearing housing by a rotational spring and a damper and rotated about a fixed origin $O_{\text {frame }}$. The developed model is limited to the machine structure of the study because the above assumptions are valid for this structure only. Detailed information about the machine structure is presented in Section V.

The rotational motion equation of the motor frame is formulated as

$$
\begin{aligned}
& \mathbf{I} \ddot{\boldsymbol{\theta}}+\mathbf{C} \dot{\boldsymbol{\theta}}+\mathbf{K} \boldsymbol{\theta}=\mathbf{T} \\
& \mathbf{I}=\left[\begin{array}{cc}
I_{\text {frame }, x} & 0 \\
0 & I_{\text {frame }, y}
\end{array}\right], \mathbf{C}=\left[\begin{array}{cc}
c_{\text {frame }, x} & 0 \\
0 & c_{\text {frame }, y}
\end{array}\right], \\
& \mathbf{K}=\left[\begin{array}{cc}
k_{\text {frame }, x} & 0 \\
0 & k_{\text {frame }, y}
\end{array}\right], \mathbf{T}=\left\{\begin{array}{l}
T_{x} \\
T_{y}
\end{array}\right\}, \\
& \boldsymbol{\theta}=\left\{\begin{array}{c}
\theta_{\text {stator }, x} \\
\theta_{\text {stator }, y}
\end{array}\right\}, \dot{\boldsymbol{\theta}}=\left\{\begin{array}{l}
\dot{\theta}_{\text {stator }, x} \\
\dot{\theta}_{\text {stator }, y}
\end{array}\right\}, \ddot{\boldsymbol{\theta}}=\left\{\begin{array}{l}
\ddot{\theta}_{\text {stator }, x} \\
\ddot{\theta}_{\text {stator }, y}
\end{array}\right\}
\end{aligned}
$$

where $\mathbf{I}$ is the mass moment of inertia of the motor frame and $\mathbf{C}$ and $\mathbf{K}$ are the rotational damping and stiffness of the motor frame, respectively. Rotational displacement, velocity, and acceleration vectors of the frame are denoted by $\boldsymbol{\theta}, \dot{\boldsymbol{\theta}}$, and $\ddot{\boldsymbol{\theta}}$. 
The moment caused by the UMP $\left(F_{x}, F_{y}\right)$ acting on the frame can be calculated as

$$
\begin{aligned}
& T_{x}=-\sum_{i=1}^{n} F_{i, y} l_{\mathrm{ump}, i} \\
& T_{y}=-\sum_{i=1}^{n} F_{i, x} l_{\mathrm{ump}, i}
\end{aligned}
$$

When the motor frame is vibrated by the moment, the displacements of the stator bore at the $i$ th node where the UMP is applied are given by

$$
x_{\text {stator }, i}=l_{\text {ump }, i} \tan \theta_{y}, y_{\text {stator }, i}=l_{\text {ump }, i} \tan \theta_{x}
$$

where $l_{\mathrm{ump}, i}$ is the axial length between the stator rotation center and the node where the eccentricity is defined. Eccentricity generated by the displacement of the stator bore can be calculated by

$$
\begin{aligned}
& e_{\text {stator }, i}(t)=\sqrt{\left(x_{\text {stator }, i}(t)\right)^{2}+\left(y_{\text {stator }, i}(t)\right)^{2}} \\
& \theta_{\text {stator }, i}(t)=\tan ^{-1}\left(\frac{y_{\text {stator }, i}(t)}{x_{\text {stator }, i}(t)}\right)
\end{aligned}
$$

\section{Combined Eccentricity}

From previous eccentricity models, the combined eccentricity of the $i$ th element of an electrically active part of the rotor is defined as in (14) and (15).

$$
e_{\mathrm{comb}, i}(t)=\left\{\begin{array}{c}
\left(e_{i, \mathrm{st}} \cos \theta_{i, \mathrm{st}}+x_{2}(t)+x_{\text {stator }, i}(t)\right)^{2} \\
+\left(e_{i, \mathrm{st}} \sin \theta_{i, \mathrm{st}}+y_{2}(t)+y_{\text {stator }, i}(t)\right)^{2}
\end{array}\right\}^{1 / 2}
$$

$$
\theta_{\mathrm{comb}, i}(t)=\tan ^{-1}\left(\frac{e_{i, \mathrm{st}} \sin \theta_{i, \mathrm{st}}+y_{2}(t)+y_{\mathrm{stator}, i}(t)}{e_{i, \mathrm{st}} \cos \theta_{i, \mathrm{st}}+x_{2}(t)+x_{\mathrm{stator}, i}(t)}\right)
$$

\section{Analytical Calculation of the UMP by ECCENTRICITY AT PMSM}

In this section, an analytical UMP model for a permanent magnet synchronous motor is described. The basic concept is to determine the air gap flux by modulating the fundamental component of the magnetomotive force (MMF) wave considering the air gap permeance and calculate the corresponding force components by the Maxwell stress tensor method.

In the model, the fundamental component of the air gap MMF is taken from the study by Chen et al. [11] using the same assumptions but with a slight modification, i.e., also the relative permeability of the permanent magnet is considered to calculate the rotor MMF as in (17). The amplitude of the fundamental

\begin{tabular}{|c|c|c|}
\hline Symbol & QUANTITY & Value \\
\hline$P$ & rated power & $30 \mathrm{~kW}$ \\
\hline$n$ & rated speed & $4100 \mathrm{rpm}$ \\
\hline$T$ & rated torque & $69.9 \mathrm{~N} \cdot \mathrm{m}$ \\
\hline$I$ & rated current & $41 \mathrm{~A}$ \\
\hline$k_{\mathrm{W}}$ & winding factor & 0.93 \\
\hline$p$ & number of pole pairs & 3 \\
\hline$m$ & number of phases & 3 \\
\hline$N$ & number of series turns per phase & 48 \\
\hline$B_{\mathrm{r}}$ & magnetic remanence & $1.07 \mathrm{~T}$ \\
\hline$h_{\mathrm{m}}$ & thickness of permanent magnet & $3.5 \mathrm{~mm}$ \\
\hline$\mu_{0}$ & air permeability & $4 \pi \times 10^{7} \mathrm{~N} / \mathrm{A}^{2}$ \\
\hline$\mu_{\mathrm{r}, \mathrm{PM}}$ & relative permeability of permanent magnet & 1.0445 \\
\hline$\alpha_{\mathrm{p}}$ & magnetic pitch/pole ratio & 0.75 \\
\hline$\delta_{0}$ & $\begin{array}{l}\text { mean air gap length including rotor surface } \\
\text { thickness }(3 \mathrm{~mm})\end{array}$ & $5.5 \mathrm{~mm}$ \\
\hline$R_{\mathrm{r}}$ & outer radius of permanent magnet rotor & $77 \mathrm{~mm}$ \\
\hline$l$ & length of permanent magnet rotor & $126 \mathrm{~mm}$ \\
\hline$\varphi$ & power factor angle & 0.142 \\
\hline$I_{\text {frame }}$ & mass moment of inertia of the frame & $4 \mathrm{~kg} \cdot \mathrm{m}^{2}$ \\
\hline$k_{\text {frame }, x}$ & rotation stiffness of the frame about $x$-axis & $1.20 \cdot 10^{6} \mathrm{~N} / \mathrm{m}$ \\
\hline$k_{\text {frame }, y}$ & rotation stiffness of the frame about $y$-axis & $7.57 \cdot 10^{5} \mathrm{~N} / \mathrm{m}$ \\
\hline$c_{\text {frame }, x}$ & rotation damping of the frame about $x$-axis & $81.81 \mathrm{~N} \cdot \mathrm{s} / \mathrm{m}$ \\
\hline$c_{\text {frame }, y}$ & rotation damping of the frame about $y$-axis & $65.09 \mathrm{~N} \cdot \mathrm{s} / \mathrm{m}$ \\
\hline
\end{tabular}
MMF of the air gap is written as

$$
\begin{gathered}
F_{\mathrm{m}}=\sqrt{F_{\mathrm{sm}}^{2}+F_{\mathrm{rm}}^{2}-2 F_{\mathrm{sm}} F_{\mathrm{rm}} \sin \varphi} \\
F_{\mathrm{rm}}=\frac{4 B_{\mathrm{r}} h_{\mathrm{m}}}{\pi \mu_{\mathrm{r}, \mathrm{PM}} \mu_{0}} \sin \left(\frac{\alpha_{\mathrm{p}} \pi}{2}\right), F_{\mathrm{sm}}=\frac{\sqrt{2} m N k_{\mathrm{w}}}{\pi p} I
\end{gathered}
$$

TABLE I

PARAMETERS OF THE ELECTRICAL MACHINE UNDER STUDY

where $F_{\mathrm{sm}}$ and $F_{\mathrm{rm}}$ are the amplitudes of the fundamental MMF waves for the stator and the permanent magnet rotor, respectively. The variables are explained in Table I. integration using the Maxwell stress over the rotor surface as ary and $\alpha$ is a variable for defining air-gap's circumferential location on the rotor surface as in Fig. 1. lished using two modeling approaches. In the first approach, the UMP is applied to the rotor model as a linear spring element. In the second approach, the UMP is applied as a nonlinear force. stiffness is first defined employing the UMP model developed by Guo et al. [9] with several assumptions. In the UMP model, if oscillating terms except the first constant term are ignored and the power series having only the first two terms as in (20) are used under the assumption $\varepsilon^{2} \ll 1$, the UMP model can be simplified as in (21) and (22).
The resulting force of the UMP can be obtained by direct

$$
\begin{aligned}
& F_{x}=\int_{0}^{2 \pi} \sigma R_{\mathrm{r}} l \cos \alpha d \alpha \\
& F_{y}=\int_{0}^{2 \pi} \sigma R_{\mathrm{r}} l \sin \alpha d \alpha
\end{aligned}
$$

Here, $\sigma$ is the Maxwell stress normal to the iron and air bound-

\section{Modeling of A Rotor-Bearing System With UMP}

In this section, the rotor-bearing model with UMP is estab-

For the simulation using the first approach, a linearized UMP

$$
\begin{gathered}
\frac{1}{1-\varepsilon^{2}} \approx 1+\varepsilon^{2}, \sqrt{1-\varepsilon^{2}} \approx 1-\frac{\varepsilon^{2}}{2} \\
F_{x}(\varepsilon, \theta)=\frac{R l \pi \mu_{0}}{2 \delta_{0}^{2}} F_{\mathrm{m}}^{2}\left(\varepsilon+\frac{5}{4} \varepsilon^{3}+\frac{5}{16} \varepsilon^{5}+\frac{1}{16} \varepsilon^{7}\right) \cos \theta
\end{gathered}
$$




$$
F_{y}(\varepsilon, \theta)=\frac{R l \pi \mu_{0}}{2 \delta_{0}^{2}} F_{\mathrm{m}}^{2}\left(\varepsilon+\frac{5}{4} \varepsilon^{3}+\frac{5}{16} \varepsilon^{5}+\frac{1}{16} \varepsilon^{7}\right) \sin \theta
$$

Moreover, if it is assumed that the eccentricity has purely horizontal or vertical direction components, i.e., the coupled terms between the $x$ and $y$ directions are ignored, the UMP stiffness $\left(k_{\mathrm{ump}, x}, k_{\mathrm{ump}, y}\right)$ can be obtained as follows. When $\theta$ is zero (pure $x$-direction eccentricity, $x=\delta_{0} \varepsilon$ ),

$$
F_{x}=\frac{R l \pi \mu_{0}}{2 \delta_{0}^{3}} F_{\mathrm{m}}^{2}\left(x+\frac{5 x^{3}}{4 \delta_{0}^{2}}+\frac{5 x^{5}}{16 \delta_{0}^{4}}+\frac{x^{7}}{16 \delta_{0}^{6}}\right)
$$

When $\theta$ is $\pi / 2$ (pure $y$-direction eccentricity, $y=\delta_{0} \varepsilon$ ),

$$
F_{y}=\frac{R l \pi \mu_{0}}{2 \delta_{0}^{3}} F_{\mathrm{m}}^{2}\left(y+\frac{5 y^{3}}{4 \delta_{0}^{2}}+\frac{5 y^{5}}{16 \delta_{0}^{4}}+\frac{y^{7}}{16 \delta_{0}^{6}}\right)
$$

Above, the UMP force is linearized as a derivative in a static eccentricity $\left(x_{\mathrm{st}}, y_{\mathrm{st}}\right)$, and the UMP stiffness is presented as

$$
\left.k_{\mathrm{ump}, x} \approx \frac{d F_{x}(x)}{d x}\right|_{x_{\mathrm{st}}},\left.k_{\mathrm{ump}, y} \approx \frac{d F_{y}(y)}{d y}\right|_{y_{\mathrm{st}}}
$$

In conclusion, the equation of motion with UMP can be established by using the UMP stiffness vector $\mathbf{K}_{\text {ump }}$ linearized at a given static eccentricity as

$$
\mathbf{M} \ddot{\mathbf{q}}+(\mathbf{C}+\Omega \mathbf{G})\left(\dot{\mathbf{q}}+\left(\mathbf{K}-\mathbf{K}_{\mathrm{ump}}\right) \mathbf{q}=\mathbf{F}_{\mathrm{ub}}+\mathbf{F}_{\mathrm{g}}\right.
$$

where $\mathbf{q}$ is the displacement vector and $\mathbf{M}, \mathbf{C}, \mathbf{G}$, and $\mathbf{K}$ are the mass, damping, gyroscopic, and stiffness matrices, respectively. Term $\Omega$ is the rotor angular velocity. Correspondingly, $\mathbf{F}_{\mathrm{ub}}$ and $\mathbf{F}_{\mathrm{g}}$ denote the unbalance force and the gravity force, respectively.

In the second method, UMP is added as an external force $\left(\mathbf{F}_{\text {ump }}\right)$ directly to the equation of motion as follows

$$
\mathbf{M} \ddot{\mathbf{q}}+(\mathbf{C}+\Omega \mathbf{G}) \dot{\mathbf{q}}+\mathbf{K q}=\mathbf{F}_{\mathrm{ub}}+\mathbf{F}_{\mathrm{g}}+\mathbf{F}_{\mathrm{ump}}
$$

\section{Simulation}

In this section, simulations using the rotor-bearing system models in Section IV are presented. The simulations were conducted for a centrifugal pump with an integrated PMSM. Results for several cases were obtained and compared to identify the effects of UMP on the vibration of the machine.

\section{A. Electrical Machine Under Study}

The machine under study consists of a motor, a bearing unit, and a volute case. It has an overhang structure in which the rotor is not supported on the motor rear side; further, the impeller is not considered. The structure of the machine is shown in Fig. 5, and the machine parameters are given in Table I. The mass unbalance of the rotor is located at the face of the nonpump side, and its magnitude is $110.25 \mathrm{~g} \cdot \mathrm{mm}$ corresponding to the balancing grade $\mathrm{G} 2.5$. Two angular contact ball bearings support the rotor, and their stiffness is estimated by using a simple method proposed by Gargiulo [17]. According to this method, the stiffness is calculated by considering the ball diameter, the ball numbers, and the bearing radial load. Here, the bearing load is estimated on the assumption of $20 \%$ static eccentricity and a

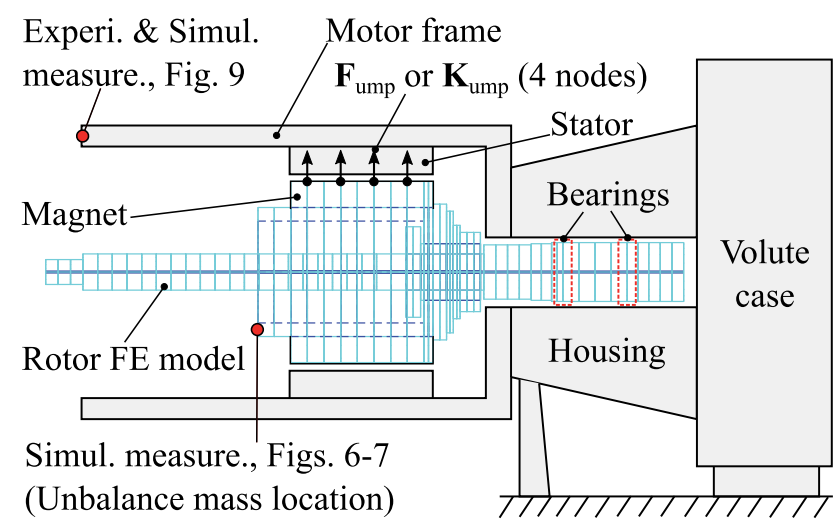

Fig. 5. Structure of the electrical machine under study.

$20 \mu \mathrm{m}$ (0-peak) rotor whirling vibration condition, because the result of the $20 \%$ eccentricity case will be compared with the experimental result. In conclusion, each bearing has constant stiffness $\left(k_{\text {bearing }}=1.4 \cdot 10^{8} \mathrm{~N} / \mathrm{m}\right)$ and damping $\left(3.5 \cdot 10^{3} \mathrm{~N} \cdot \mathrm{s} / \mathrm{m}\right)$ in both the horizontal and vertical directions. Here, damping is estimated as $2.5 \cdot 10^{-5} \cdot k_{\text {bearing }}$ based on the suggestion presented in [18].

\section{B. Simulation Method}

For the rotordynamic analysis, the rotor is modeled with beam finite elements that have four degrees of freedom per node. This model assumes that there is no displacement in the axial direction and no rotation around the rotor axis. To consider axial-varying static eccentricity, the electrically active part of the rotor is divided into four parts and individual static eccentricity is applied to each part. In this study, seven cases in total are simulated; without UMP, with UMP in three conditions of eccentricity (mixed and axial-varying eccentricity with $0-0 \%, 10-0 \%$ and $20-0 \%$ static eccentricities), and three cases with UMP including frame vibration. The axial-varying static eccentricity is expressed as initial side eccentricity and final side eccentricity. The direction angle of the static eccentricity is set to zero in all cases. The static eccentricities of the divided rotor parts are calculated using (3) to (6).

For the simulation using the linear spring model of the UMP, the UMP stiffness is calculated using the process presented in Section IV. To consider axial-varying static eccentricity, the individual UMP stiffness is calculated at the obtained static eccentricity of each rotor part and applied to the node of the rotor part. Total UMP stiffness values in the horizontal direction for the entire rotor are calculated as $1.399 \cdot 10^{6}$ for $0-0 \%, 1.403 \cdot 10^{6}$ for $10-0 \%$, and $1.414 \cdot 10^{6} \mathrm{~N} / \mathrm{m}$ for $20-0 \%$ static eccentricity. The vertical direction stiffness is the same $1.399 \cdot 10^{6} \mathrm{~N} / \mathrm{m}$ for all cases because there is no eccentricity in the vertical direction. The motion equation is defined as an eigenvalue problem and the critical speeds are calculated by solving this problem. However, the motor frame vibration model cannot be included in this method.

In the simulation applying the external force model of the UMP, a time transient analysis using numerical integration is 


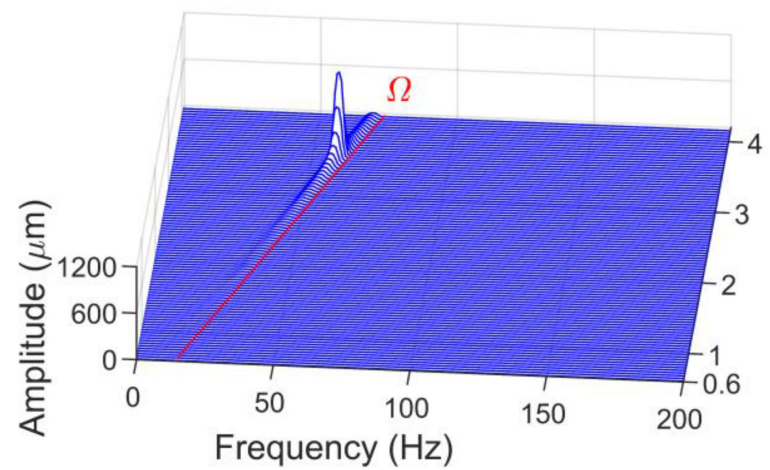

(a) Without UMP

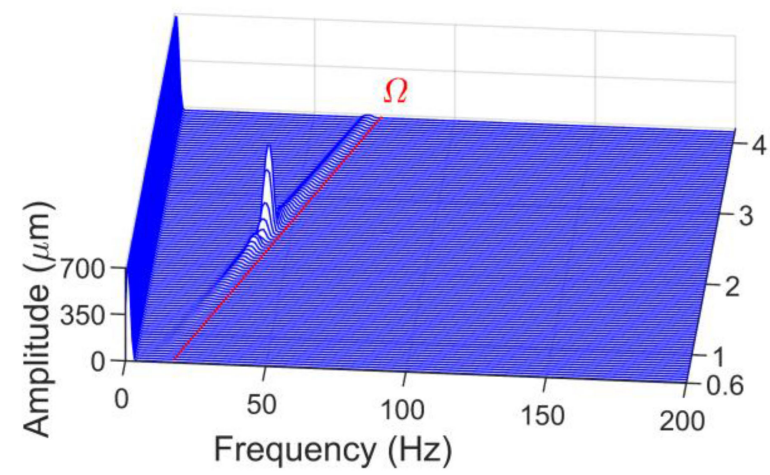

(c) With UMP (mixed eccentricity with $20-0 \%$ static eccentricity)

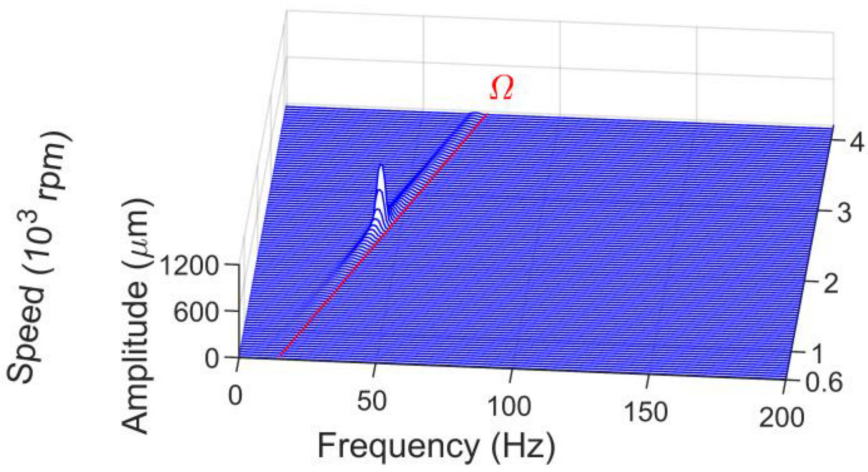

(b) With UMP (only dynamic eccentricity)

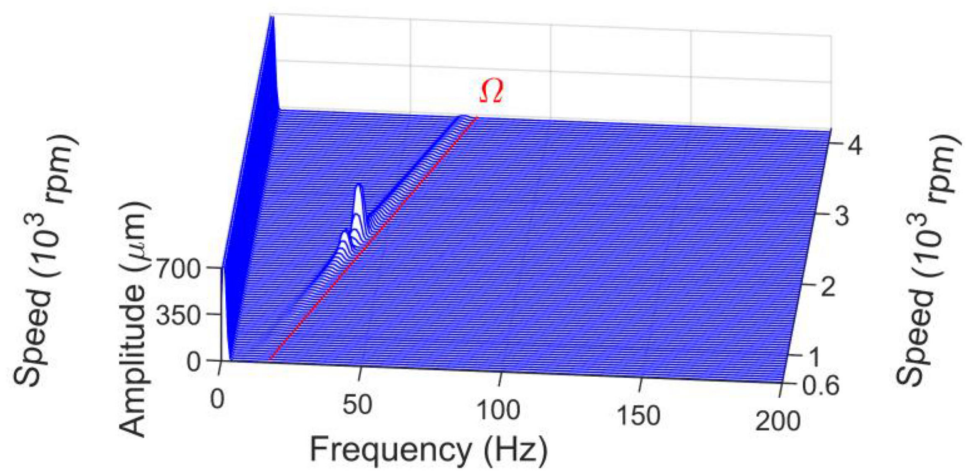

(d) With UMP \& frame model (mixed eccentricity with 20-0\% static eccentricity)

Fig. 6. 3D spectral map of horizontal direction displacement $(\mu \mathrm{m})$ at the unbalance mass location to determine a change in critical speeds.

conducted to solve the motion equation. The numerical integration is performed using the ode $15 \mathrm{~s}$ function in MATLAB. In the analysis process, the rotor location is updated at every time step and the UMP is calculated using eccentricity from the updated rotor location. For the simulation including frame vibration, the inertia, stiffness, and damping of the frame presented in Table I are used. These values were predicted by using two natural frequencies $(74$ and $93 \mathrm{~Hz})$ related to the frame's rotational vibration modes determined by experimental measurement [16]. To investigate the UMP effects, a ramp from 10 to $70 \mathrm{~Hz}$ in $60 \mathrm{~s}$ is simulated for all cases.

\section{Simulation Results}

From the simulation using the linear spring model, critical speeds of the rotor are obtained for four cases without frame vibration. Again, $3 \mathrm{D}$ spectral maps of the rotor vibration were obtained from the simulation using the external force model. Here, displacement vibration is measured at the rotor node where the unbalance mass is located as in Fig. 5., because rotor vibration can be only measured when not including the frame model. For a comparison with the experimental results, in the case including the frame model, also velocity is measured at the same frame location with the experimental measurement, which is presented in Section IV on experimental verification.
The $3 \mathrm{D}$ spectral maps for the four cases: without UMP, with UMP at $0-0 \%$ and $0-20 \%$ axial-varying static eccentricity, and with UMP including frame vibration at $20-0 \%$ axial-varying static eccentricity are presented in Figs. 6 and 7. From the peak point of the 3D spectral map, the first backward/forward critical speeds can be found. Changes in the critical speeds caused by the UMP are detected and presented in Table II. The negative stiffness effect was observed similarly in both simulation methods. The critical speeds were decreased by the UMP, and this effect was slightly amplified by frame vibration. The effect of static eccentricity on the negative stiffness was small in comparison with the effect of dynamic eccentricity. This finding is in agreement with the calculation result indicating that the UMP stiffness change from the static eccentricity is small.

Moreover, the effect of static eccentricity on the negative stiffness was shown with only a little difference between two methods. In the method using the linear spring model of the UMP, the effect by static eccentricity appeared to be smaller than in the other method. Furthermore, it is shown that the excitation of the backward whirling mode increases when the static eccentricity increases as shown in Fig. 6. The backward whirling mode is excited by the anisotropic support stiffness [19], which is amplified by the anisotropic negative stiffness caused by static eccentricity.

To determine the additional excitations from the UMP, 3D spectral maps with a decreased range of the $\mathrm{z}$ axis are presented 


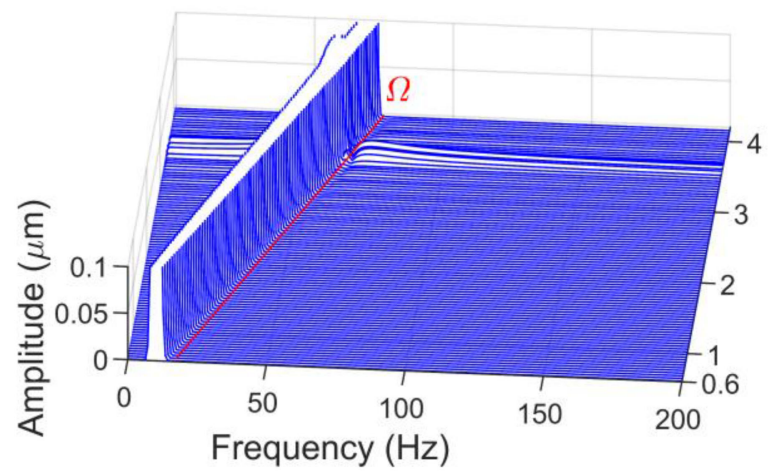

(a) Without UMP

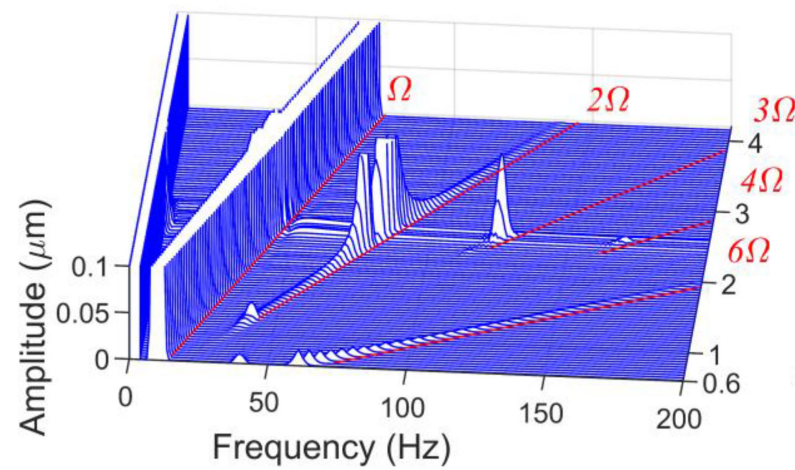

(c) With UMP (mixed eccentricity with $20-0 \%$ static eccentricity)

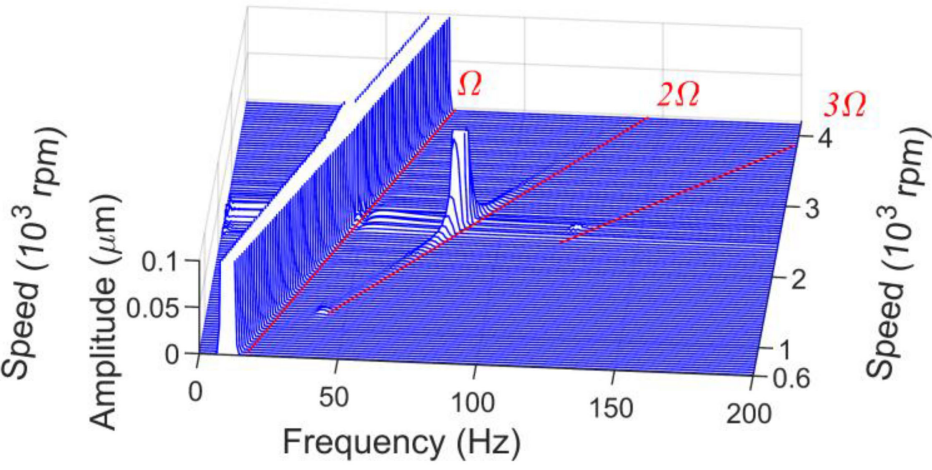

(b) With UMP (only dynamic eccentricity)

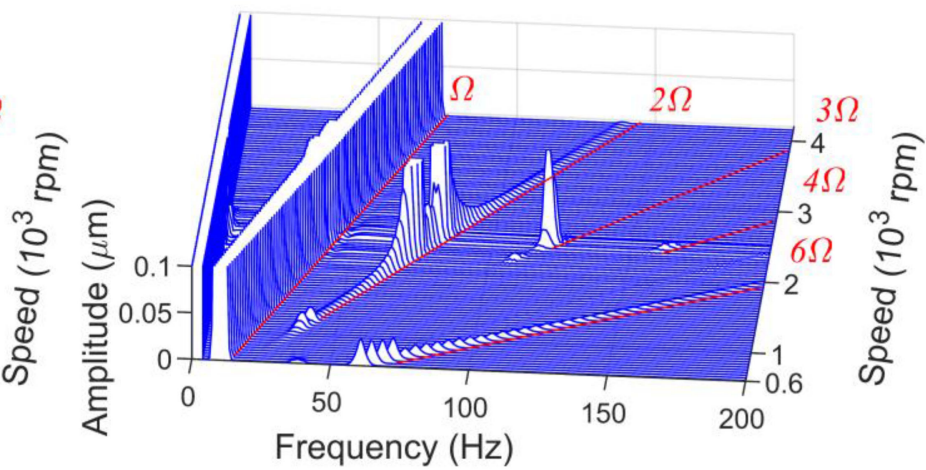

(d) With UMP \& frame model (mixed eccentricity with $20-0 \%$ static eccentricity)

Fig. 7. 3D spectral map of horizontal direction displacement $(\mu \mathrm{m})$ at unbalance mass location to determine higher frequency excitations.

TABLE II

Change in CRitical SpeEds (Simulation 1: Linear SpRing Model, Simulation 2: NONLINEAR ForCE MODEL)

\begin{tabular}{llll}
\hline \hline \multirow{2}{*}{ Method } & \multicolumn{1}{c}{ CASE } & $\begin{array}{c}\text { 1ST BW } \\
\text { CRITICAL } \\
\text { SPEED, Hz }\end{array}$ & $\begin{array}{c}\text { 1ST FW } \\
\text { CRITICAL } \\
\text { SPEED, Hz }\end{array}$ \\
\hline \multirow{3}{*}{$\begin{array}{l}\text { Simulation1 } \\
\text { (Eigenvalue) }\end{array}$} & Without UMP & 54.1 & 59.1 \\
& With UMP (0-0\%) & $38.6(\downarrow 15.5)$ & $42.2(\downarrow 16.9)$ \\
& With UMP (10-0\%) & $38.6(\downarrow 15.5)$ & $42.1(\downarrow 17.0)$ \\
& Without UMP & $\left.-{ }^{*}\right)$ & $59.0(\downarrow 17.1)$ \\
& With UMP (0-0\%) & $42.2(\downarrow 11.9)$ & $43.8(\downarrow 15.9)$ \\
& With UMP (10-0\%) & $38.2(\downarrow 15.9)$ & $42.2(\downarrow 17.5)$ \\
Simulation2 & With UMP (20-0\%) & $37.4(\downarrow 16.7)$ & $41.4(\downarrow 18.3)$ \\
(Numerical & With UMP (0-0\%) & $37.4(\downarrow 16.7)$ & $41.4(\downarrow 18.3)$ \\
time & \& frame vibration & & \\
integration) & With UMP (10-0\%) & $37.4(\downarrow 16.7)$ & $41.4(\downarrow 18.3)$ \\
& \& frame vibration & & \\
& With UMP (20-0\%) & $36.6(\downarrow 17.5)$ & $39.8(\downarrow 19.9)$ \\
\hline \hline
\end{tabular}

${ }^{*}$ Backward mode (without UMP case) is not excited in Simulation 2, and therefore, the effect of UMP is found by comparing with the critical speed $(54.1 \mathrm{~Hz})$ in Simulation 1. in Fig. 7. It is found that harmonics with $2 \Omega, 3 \Omega, 4 \Omega$, $5 \Omega$, and $6 \Omega$ (2.line frequency) frequencies are generated, and their amplitudes increase when the static eccentricity increases. In particular, it is shown that the $6 \Omega$ frequency component occurs especially when static eccentricity is present. Finally,
TABLE III

VIBRATION FREQUENCIES IN SIMULATION RESULT WITH UMP AS AN EXTERNAL NONLINEAR FORCE

\begin{tabular}{ll}
\hline \hline \multicolumn{1}{c}{ CASE } & \multicolumn{1}{c}{ MAIN OCCURRENCE FREQ. } \\
\hline Without UMP & $\Omega$ \\
With UMP $(0-0 \%$ static $)$ & $\Omega, 2 \Omega$ \\
With UMP $(10-0,20-0 \%$ static $)$ & $\Omega, 2 \Omega, 6 \Omega\left(2 \cdot\right.$ line freq. $\left.{ }^{*}\right)$ \\
\hline \hline
\end{tabular}

${ }^{*}$ Line freq. $=p \Omega=3 \Omega, p$ : pole pair no.
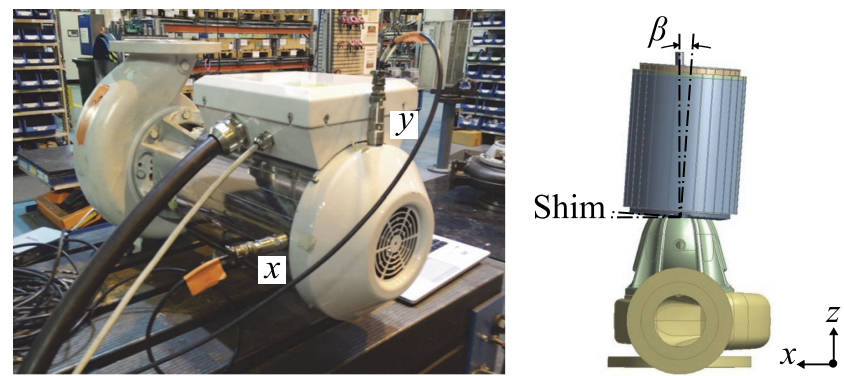

Fig. 8. Pump test rig, (left) two accelerometers for measurement, (right) axialvarying static eccentricity by shim.

it is observed that the UMP effect is slightly amplified by frame vibration. The critical speeds are lower, and especially the excitations that meet the frame natural frequency are significantly amplified. 


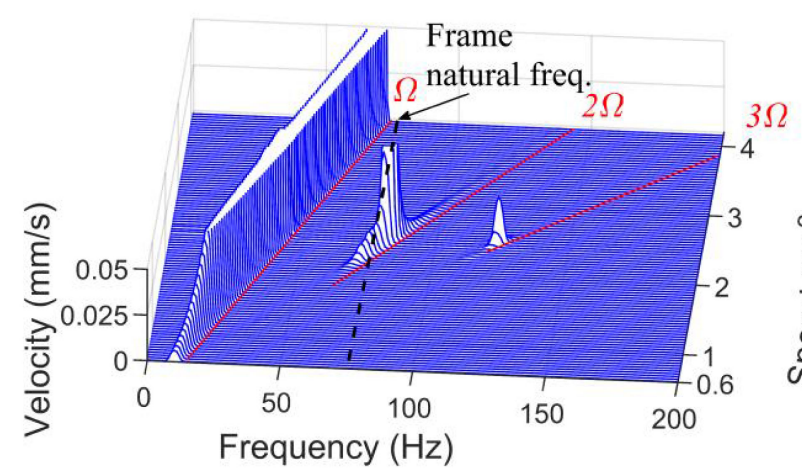

(a) Simulation, $0-0 \%$ axial-varying static eccentricity

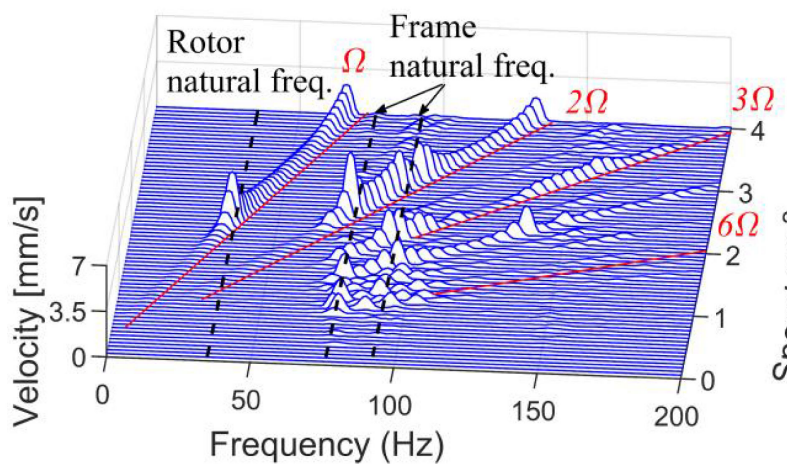

(c) Experiment, $0-0 \%$ axial-varying static eccentricity

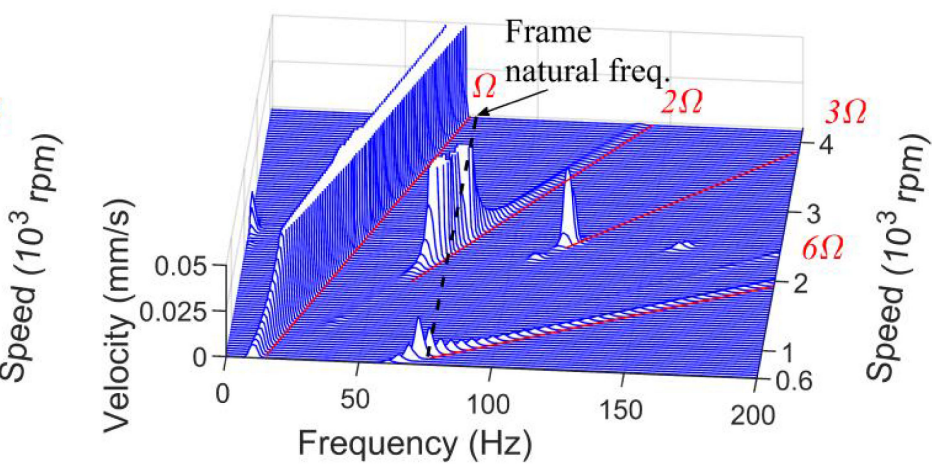

(b) Simulation, $20-0 \%$ axial-varying static eccentricity

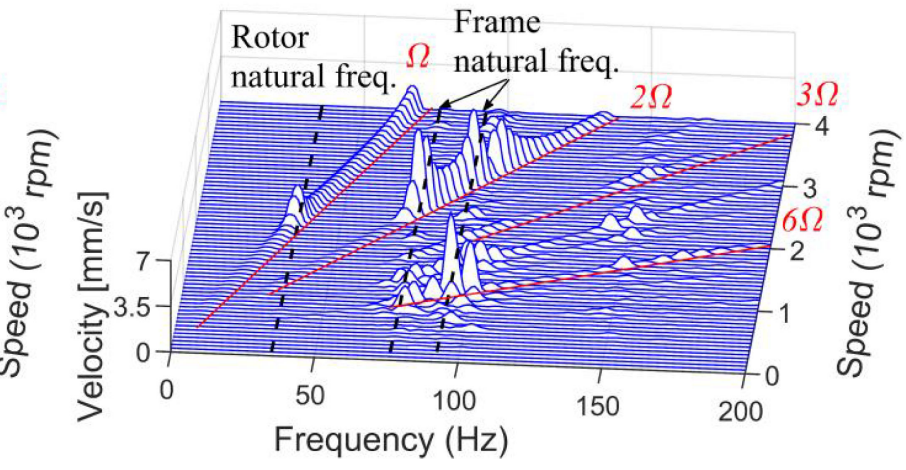

(d) Experiment, $20-0 \%$ axial-varying static eccentricity

Fig. 9. Comparison between the 3D spectral maps for the simulation and the experimental results of horizontal direction velocity (mm/s) ( $\Omega$ : rotating speed).

\section{EXPERIMENTAL VERIFICATION}

To verify the simulation results, experiments were carried out with a pump test rig. Axial-varying static eccentricity was adjusted by placing a shim between the bearing housing and the motor frame as in Fig. 8. The eccentricity was checked with a customized feeler gauge in which the measurement limits were set at $0.25 \mathrm{~mm}$ increments. For the vibration measurement, two accelerometers (IMI VO-622) were attached to the nonpump side of the motor frame in the $x$ and $y$-directions as in Fig. 8 .

A ramp from 0 to $4100 \mathrm{rpm}$ in $70 \mathrm{~s}$ was driven and 3D spectral maps for the velocity vibration of the frame were obtained as a result. In this experiment, only the cases including UMP were investigated because it is impossible to drive the rotor without the effect of the UMP. Therefore, higher frequency excitations from the UMP and the effect caused by the variation of the static eccentricity can be seen from the experimental results, but the negative stiffness effect from the presence of the UMP cannot be accurately verified. 3D spectral maps for vibration in horizontal direction for two cases with $0-0 \%$ and $20-0 \%$ axial-varying static eccentricities are presented in Fig. 9 with simulation results measured at the same location using the same vibration unit.

The experimental results show that harmonic excitations occur similarly to the simulation result, and these components are amplified at frame natural frequencies. As mentioned above in the frame vibration modeling section, the whole frame has two natural frequencies ( 73 and $93 \mathrm{~Hz}$ ) within the operating speed range. However, in simulation results presented in Fig. 9, the amplification is shown at only first natural frequency, of which the mode shape is related with vibration in horizontal direction. Moreover, it is shown that the $2 \Omega$ and $6 \Omega$ frequency components are significantly amplified by the static eccentricity. These results are in agreement with the simulation results. However, the amplitudes of these components are much higher than the ones found in the simulation results. This discrepancy is probably due to the simplifications made in the modeling of frame vibration; the topic, however, requires further study.

\section{CONCLUSION}

An eccentricity model including mixed eccentricity and axialvarying eccentricity considering frame vibration was developed. Based on the eccentricity model, analyical unbalanced magnetic pull (UMP) model was applied to the rotor simulation model of a centrifugal pump with a PMSM. From simulation results for several cases, the vibration effects caused by the UMP were studied, and the results were verified by an experimental analysis of a pump test rig. 
The key findings of the study on the effect of UMP on vibration and conclusions about the simulations can be summarized as follows.

1) The negative stiffness effect caused by the UMP decreases rotor critical speeds. This effect is mainly due to dynamic eccentricity and slightly amplified by static eccentricity and frame vibration. Moreover, the anisotropy of static eccentricity amplifies the excitation of the backward whirling mode.

2) Additional vibration components with frequencies of $2 \Omega$, $6 \Omega$ (2.line frequency), and other speed multiple frequencies are generated by the UMP. The vibration component of the $2 \cdot$ line frequency is mainly generated when static eccentricity is present, and its amplitude is increased when the static eccentricity increases.

3) The simulation method using a linear negative spring model of UMP can be used to estimate the effect of negative stiffness on rotor critical speeds. However, the method applying UMP as an external force can only be used for prediction of higher frequency excitations produced by UMP.

4) A comparison between the simulations and the experimental results showed that the proposed simulation model can be used to predict electromagnetic excitations caused by UMP from air gap eccentricity.

5) In the experimental results, the amplitude of the electromechanical excitation at the natural frequency of the frame was significantly higher than in the simulations. This difference is probably due to the assumptions used in the modeling of the frame vibration. Hence, further studies on the modeling of the frame vibration effects are required.

\section{REFERENCES}

[1] A. J. Pina Ortega and L. Xu, "Investigation of effects of asymmetries on the performance of permanent magnet synchronous machines," IEEE Trans. Energy Convers., vol. 32, no. 3, pp. 1002-1011, Sep. 2017.

[2] Z. Q. Zhu, D. Ishak, D. Howe, and J. Chen, "Unbalanced magnetic forces in permanent-magnet brushless machines with diametrically asymmetric phase windings," IEEE Trans. Ind. Appl., vol. 43, no. 6, pp. 1544-1553, Nov./Dec. 2007.

[3] Z. J. Liu and J. T. Li, "Accurate prediction of magnetic field and magnetic forces in permanent magnet motors using an analytical solution," IEEE Trans. Energy Convers., vol. 23, no. 3, pp. 717-726, Sep. 2008.

[4] M. Donát, "Computational modelling of the unbalanced magnetic pull by finite element method," Procedia Eng., vol. 48, pp. 83-89, 2012.

[5] D. G. Dorrell, W. T. Thomson, and S. Roach, "Analysis of airgap flux, current, and vibration signals as a function of the combination of static and dynamic airgap eccentricity in 3-phase induction motors," IEEE Trans. Ind. Appl., vol. 33, no. 1, pp. 24-34, Jan./Feb. 1997.

[6] D. G. Dorrell, "Sources and characteristics of unbalanced magnetic pull in three-phase cage induction motors with axial-varying rotor eccentricity," IEEE Trans. Ind. Appl., vol. 47, no. 1, pp. 12-24, Jan./Feb. 2011.

[7] Y. Li and Z. Q. Zhu, "Cogging torque and unbalanced magnetic force prediction in PM machines with axial-varying eccentricity by superposition method," IEEE Trans. Magn., vol. 53, no. 11, Nov. 2017, Art. no. 1400404.

[8] A. Tenhunen, T. Benedetti, T. P. Holopainen, and A. Arkkio, "Electromagnetic forces of the cage rotor in conical whirling motion," IEE Proc.Electr. Power Appl., vol. 150, no. 5, pp. 563-568, Sep. 2003.

[9] D. Guo, F. Chu, and D. Chen, "The unbalanced magnetic pull and its effects on vibration in a three-phase generator with eccentric rotor," J. Sound Vib., vol. 254, no. 2, pp. 297-312, 2003.

[10] C. Di, X. Bao, H. Wang, Q. Lv, and Y. He, "Modeling and analysis of unbalanced magnetic pull in cage induction motors with curved dynamic eccentricity," IEEE Trans. Magn., vol. 51, no. 8, p. 8106507, Aug. 2015.
[11] X. Chen, S. Yuan, and Z. Peng, "Nonlinear vibration for PMSM used in HEV considering mechanical and magnetic coupling effects," Nonlinear Dyn., vol. 80, no. 1-2, pp. 541-552, 2015.

[12] C. Xiang, F. Liu, H. Liu, L. Han, and X. Zhang, "Nonlinear dynamic behaviors of permanent magnet synchronous motors in electric vehicles caused by unbalanced magnetic pull," J. Sound Vib., vol. 371, pp. 277-294, 2016.

[13] P. Lošák and R. Vlach, "Study of the response of the shaft loaded by unbalanced magnetic pull," in Proc. 16th Int. Conf. Mechatronics, Mechatronika, 2014, pp. 79-84.

[14] X. Xu, Q. Han, and F. Chu, "Nonlinear vibration of a generator rotor with unbalanced magnetic pull considering both dynamic and static eccentricities," Arch. Appl. Mech., vol. 86, no. 8, pp. 1521-1536, 2016.

[15] P. Pennacchi, "Computational model for calculating the dynamical behaviour of generators caused by unbalanced magnetic pull and experimental validation," J. Sound Vib., vol. 312, no. 1-2, pp. 332-353, 2008.

[16] A. Posa, "Vibration behavior of a centrifugal pump with integrated permanent magnet motor," M.S. thesis, Dept. Mech. Eng., Lappeenranta Univ. Technol., Lappeenranta, Finland, 2016.

[17] E. P. J. Gargiulo, "A simple way to estimate bearing stiffness," Mach. Des., vol. 52, no. 17, pp. 107-110, 1980.

[18] E. Krämer, Dynamics of Rotors and Foundations. Berlin Heidelberg, Germany: Springer-Verlag GmbH, 1993.

[19] L. M. Greenhill and G. A. Cornejo, "Critical speeds resulting from unbalance excitation of backward whirl modes," in Proc. Des. Eng. Tech. Conf., vol. 84-2, 1995, pp. 991-1000.

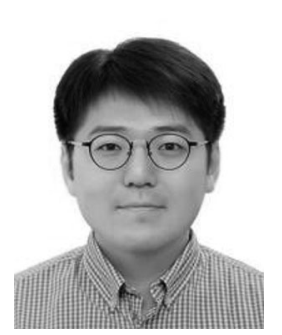

Heesoo Kim was born in Seoul, Korea, in 1979. He received the B.S. and M.S. degrees in mechanical engineering from Hanyang University, Seoul, Korea, in 2005 and 2007, respectively. After graduation, he worked as a Turbocharger Development Engineer for marine and vehicle engines. From 2017, he is working toward the Ph.D. degree at the Department of Mechanical Engineering, LUT University, Lappeenranta, Finland. His research interests include rotordynamics for electrical machines, specifically study of electromechanical interaction from air gap eccentricity, stator deformation, and other geometric non-idealities.

494 495

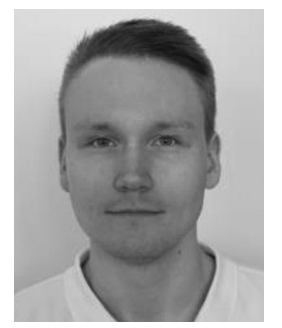

Atte Posa received the M.Sc. degree in mechanical engineering from LUT University, Finland, in 2016. After the master's studies, he started working as Product Development Engineer in Sulzer Pumps Finland Oy, Kotka, Finland. His work as Product Development Engineer is focused on mechanical seals and sealing systems.

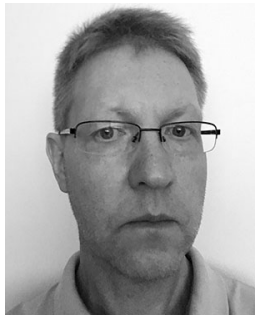

Janne Nerg (M'99-SM'12) received the M.Sc degree in electrical engineering, the Licentiate of Science (Technology) degree, and the D. Sc. (Technology) degree from LUT University, Lappeenranta, Finland, in 1996, 1998, and 2000, respectively. He is currently an Associate Professor with the Department of Electrical Engineering at LUT University. His research interests include electrical machines and drives, especially electromagnetic and thermal modeling and design of electromagnetic devices. 


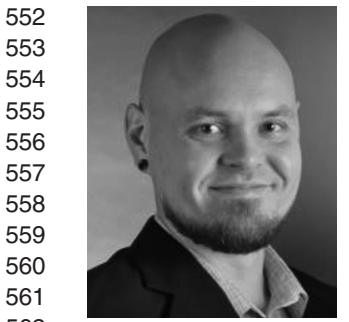

Janne Heikkinen received the M.Sc. degree in mechanical engineering from LUT University, Lappeenranta, Finland, in 2010. After the master's studies, he started his research career as a Ph.D. student at LUT University. He successfully defended his Doctoral Dissertation and received the D.Sc. degree from LUT University in 2014. He is currently working as a Postdoctoral Researcher in Laboratory of Machine Dynamics at LUT University. His research interests include rotating electric machines, especially highspeed machinery. His main expertise is in rotordynamics, structural vibrations, and vibration measurements.

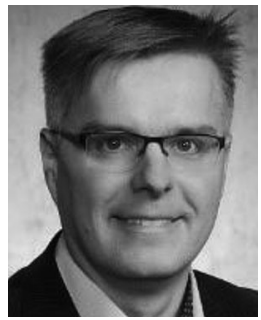

Jussi T. Sopanen (M'14) was born in 1974 in Enonkoski, Finland. He received the M. Sc. degree in mechanical engineering and the D. Sc. (Technology) degree from LUT University, Lappeenranta, Finland, in 1999 and 2004, respectively. He has been a Researcher with the Department of Mechanical Engineering at LUT University during 1999-2006. He has also worked as a Product Development Engineer in electric machine manufacturer, Rotatek Finland Ltd., from 2004 to 2005. During 2006-2012, he worked as Principal Lecturer in mechanical engineering and Research Manager with the Faculty of Technology in Saimaa University of Applied Sciences, Lappeenranta, Finland. He is currently serving as a Professor in Machine Dynamics Lab at LUT University. His research interests include rotordynamics, multi-body dynamics, and mechanical design of electrical machines. 


\title{
Analysis of Electromagnetic Excitations in an Integrated Centrifugal Pump and Permanent Magnet Synchronous Motor
}

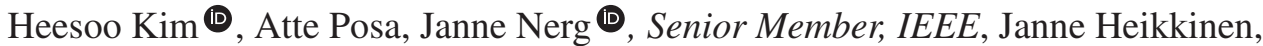 \\ and Jussi T. Sopanen $\mathbb{D}$, Member, IEEE
}

\begin{abstract}
The effect of unbalanced magnetic pull (UMP) caused by air gap eccentricity on the vibration of a permanent magnet synchronous motor (PMSM) is investigated. The force model is established analytically by the Maxwell stress method. For accurate consideration of the eccentricity condition, mixed eccentricity, axial-varying eccentricity, and eccentricity caused by motor frame vibration are modeled and combined. The model of the rotorbearing system, which includes the UMP model, is developed with two different methods. In the first method, UMP is added as a linear negative spring to the rotor model, whereas in the second method, UMP is included as an external force. The rotor system of a centrifugal pump driven by an integrated PMSM is modeled using beam elements, and the two distinct modeling approaches for UMP are applied. From the results, the UMP effect on vibration and the difference between the two modeling methods are investigated. To verify the results of the analysis, experimental work is done with a pump test rig, and results of frequency spectra are obtained. Based on the analyses and experimental work, the negative stiffness effect and additional vibration excitations caused by UMP are examined.
\end{abstract}

Index Terms-Axial-varying eccentricity, Eccentricity by frame vibration, Permanent magnet synchronous motor, Mixed eccentricity, Unbalanced magnetic pull.

$\mathbf{E}$ LECTROMECHANICAL interaction in rotating electrical machines is a significant factor in the generation of nonlinear dynamic behavior of a system. In machines with a small air gap, such nonlinear dynamic behavior can be dangerous for the rotor system, and therefore, many studies have been conducted on unbalanced magnetic pull (UMP) caused by electromechanical interaction.

The topic of UMP has been addressed in numerous studies covering various factors such as asymmetry of rotor and stator, rotor eccentricity, and magnetic saturation. Ortega et al. [1]

Manuscript received July 6, 2018; revised May 28, 2019; accepted July 15, 2019. This work was supported by LUT Doctoral School Funding. Paper no. TEC-00728-2018. (Corresponding author: Heesoo Kim.)

H. Kim, J. Heikkinen, and J. T. Sopanen are with the Department of Mechanical Engineering, LUT University, 53850 Lappeenranta, Finland (e-mail: heesoo.kim@lut.fi; janne.heikkinen@lut.fi; jussi.sopanen@lut.fi).

A. Posa is with the Sulzer Pumps Finland Oy, 48600 Kotka, Finland (e-mail: atte.posa@sulzer.com).

J. Nerg is with the Department of Electrical Engineering, LUT University, 53850 Lappeenranta, Finland (e-mail: janne.nerg@lut.fi).

Color versions of one or more of the figures in this article are available online at http://ieeexplore.ieee.org.

Digital Object Identifier 10.1109/TEC.2019.2935785 conducted experiments and a finite element analysis (FEA) to study the effects of asymmetries caused by manufacturing tolerances of the stator and rotor magnets on the performance of a PMSM. Zhu et al. [2] developed a general analytical model to predict UMP in a permanent magnet brushless AC and DC machines having a diametrically asymmetric disposition of slots and phase windings. Liu et al. [3] developed an analytical model including the effect of interaction between the pole transitions and the slot openings to analyze permanent magnet motors with a slotted stator core. The rotor eccentricity, in particular, has been widely investigated as a factor contributing to the UMP. Donat [4] calculated UMP caused by air gap eccentricity based on an electromagnetic-coupled field analysis in the Ansys software. Dorrell et al. [5] considered a combination of static and dynamic eccentricities and [6] studied a method for calculating UMP in cage induction motors; the model includes magnetic saturation and axial variation with static or dynamic eccentricity. Li et al. [7] modeled axial-varying eccentricity by a superposition method and verified the model by comparing its results with 3D FEA results. Tenhunen et al. [8] investigated UMP in an induction motor when the rotor is in whirling motion by using a method based on the principle of virtual work and measured it for a test motor supported by active magnetic bearings. Guo et al. [9] obtained analytical expressions of UMP by air gap eccentricity for any pole pair number. Di et al. [10] modeled the curved dynamic eccentricity caused by a bent rotor.

The effect of UMP has been investigated by studying the dynamic behavior of a rotor system. Chen et al. [11] studied the analytical UMP calculation method considering the magnetomotive force (MMF) of the rotor and the stator in a PMSM. They discussed the stability of the steady response by using an eigenvalue analysis for the Jeffcott rotor. Xiang et al. [12] studied the stiffness characteristics and nonlinear dynamic behavior of the Jeffcott rotor system of a PMSM affected by UMP. Losak et al. [13] modeled UMP as a spring element and investigated the rotor deflection and critical speed. Xu et al. [14] examined a rotor model considering both static and dynamic eccentricity. They compared the results with a case including dynamic eccentricity only and found that vibration displacement is increased and the rotor shaft orbit is no longer centrosymmetric and is only axisymmetric in the direction of the static eccentricity. Pennacchi [15] studied a UMP model based on the actual position of the rotor not limited to circular orbits and validated the proposed 
model by measuring the dynamical behavior of a steam turbo generator.

The rotor eccentricity is an important cause of UMP and simultaneously, a result of various factors, such as manufacturing tolerances, faults, and rotor whirling motion. Therefore, accurate modeling of eccentricity is a prerequisite for a UMP study. In most previous studies, the factors causing the rotor eccentricity have been studied individually and the effect of UMP has been investigated for a Jeffcott rotor system. In actual systems, however, different eccentricity-generating factors occur simultaneously and the total eccentricity is a result of complex interactions. Therefore, in this study, a combined model with mixed eccentricity, axial-varying eccentricity, and eccentricity caused by motor frame vibration is first developed and the UMP model with combined eccentricity is then applied to a rotor-bearing model for an actual prototype electrical machine with two different approaches, and its effects are studied. The results are verified by a comparison with experimental results for a pump test rig.

\section{ECCENTRICITY MODELING}

In the conventional analysis, two special cases of whirling motion, i.e., static eccentricity and dynamic eccentricity, are typically studied as air gap eccentricity. In static eccentricity, the whirling frequency is zero and the eccentricity results from manufacturing tolerances, wear, and misalignment of bearings. In dynamic eccentricity, the whirling frequency is equal to the rotation speed of the rotor, and the center axis of whirling motion is the same as the center axis of the stator. Such eccentricity typically results from a bent shaft or unbalance mass of the rotor.

In a real system, eccentricity is a result of a combination of both static and dynamic eccentricities and not identical in the axial direction of an eccentric rotor. Moreover, eccentricity is affected by frame vibration. To demonstrate this condition, these eccentricities are modeled individually and combined.

\section{A. Mixed Eccentricity}

Under the assumption that the rotor and the stator are ideal cylinders and the stator is rigid and does not vibrate, air gap eccentricity can be defined in the form of mixed eccentricity by combining the static and dynamic eccentricities. Mixed eccentricity can be explained as a condition in which the rotor rotates with a certain whirling amplitude about the eccentric axis, which is displaced from the center axis of the stator bore, i.e., eccentricity between the stator bore and the bearing bore center is given as a static condition, and the rotor rotates about the bearing bore center with a whirling amplitude. Therefore, mixed eccentricity can be defined as a displacement vector of the rotor center with the stator bore center as the initial point. It is dependent on time and expressed as magnitude $e_{0}$ and direction angle $\theta_{0}$.

To model this condition, two reference coordinate systems are defined as in Fig. 1. The origin $O_{1}$ of the $x_{1}-y_{1}$ coordinate system is the geometric center of the stator, and the origin $\mathrm{O}_{2}$ of the $x_{2}-y_{2}$ coordinate system is the center of rotor whirling and can also be regarded as the geometric center of the bearings. $O_{\mathrm{r}}$ is the geometric center of the rotor. The $x_{2}-y_{2}$ coordinate

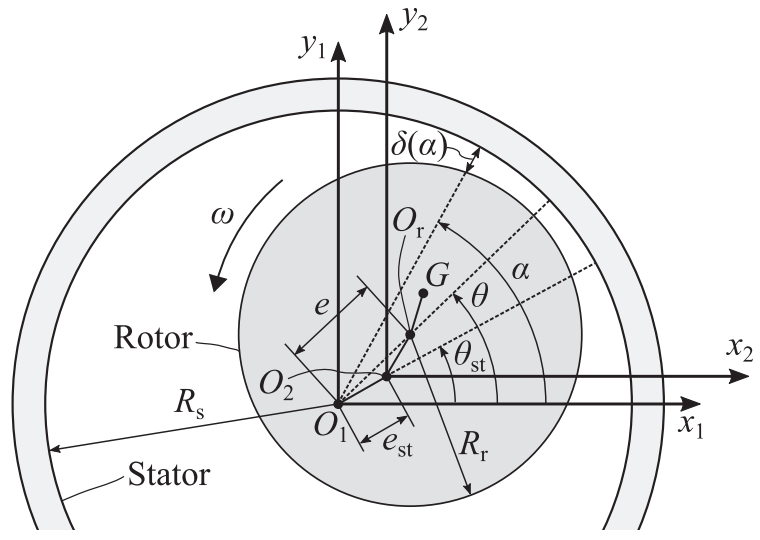

Fig. 1. Cross-sectional view of an eccentric rotor. ( $G$ : rotor mass center).

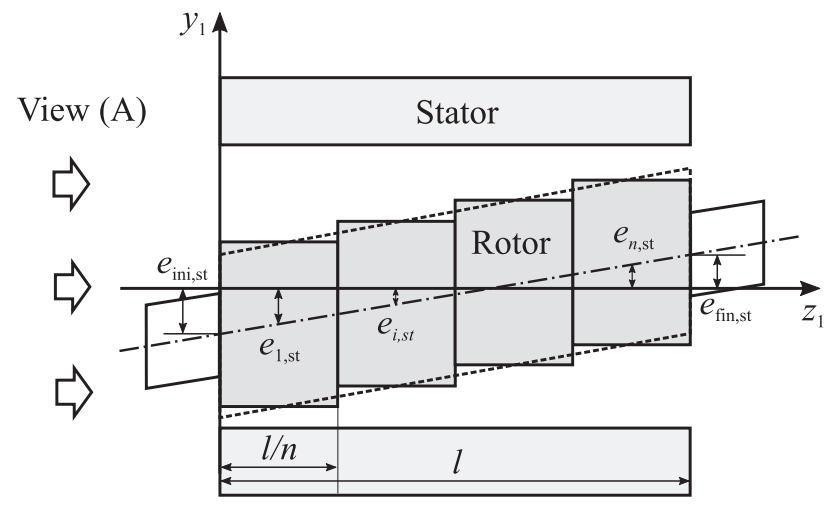

Fig. 2. Finite element model representation of an axial-varying static eccentric rotor (dotted line: real rotor condition).

system is translated parallel to the $x_{1}-y_{1}$ coordinate system as the same degree as the static eccentricity. Therefore, the rotor displacement can be defined as $x_{2}$ and $y_{2}$ coordinates of the rotor center $O_{\mathrm{r}}$, and the mixed eccentricity can be defined by combining static eccentricity and rotor displacement. Therefore, the magnitude and direction angle of mixed eccentricity are defined as

$$
\begin{aligned}
& e(t)=\sqrt{\left(e_{\mathrm{st}} \cos \theta_{\mathrm{st}}+x_{2}(t)\right)^{2}+\left(e_{\mathrm{st}} \sin \theta_{\mathrm{st}}+y_{2}(t)\right)^{2}} \\
& \theta(t)=\tan ^{-1}\left(\frac{e_{\mathrm{st}} \sin \theta_{\mathrm{st}}+y_{2}(t)}{e_{\mathrm{st}} \cos \theta_{\mathrm{st}}+x_{2}(t)}\right)
\end{aligned}
$$

where $e_{\mathrm{st}}$ and $\theta_{\mathrm{st}}$ are the amplitude and the direction angle of static eccentricity, respectively. The coordinates $x_{2}$ and $y_{2}$ denote the instantaneous displacements of the rotor center.

\section{B. Axial-Varying Eccentricity}

In an actual system, air gap eccentricity is not consistent with the axial direction because of manufacturing tolerances and assembly misalignment. On the other hand, for calculation of UMP, it must be assumed that the air gap is consistent with the axial direction. Because the magnitude of the UMP is proportional to the axial length of the electrical active rotor, the rotor can be modeled as divided elements having axially consistent and individual eccentricity, as in Fig. 2. To define the mixed eccentricity in this finite element model, the static 


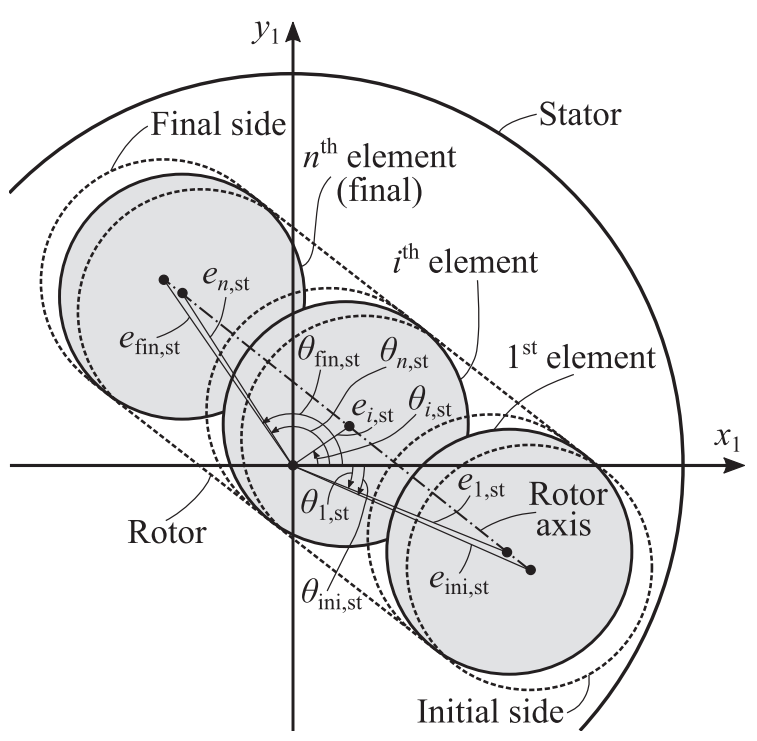

Fig. 3. View (A) of the finite element model with axial-varying static eccentricity. eccentricities $\left(e_{i, \mathrm{st}}, \theta_{i, \mathrm{st}}\right)$ for all elements of the rotor have to be determined. If the rotor is not bent, the static eccentricity of an arbitrary intermediate rotor element can be calculated from the eccentricities of the initial and final side using the geometric relationship presented in Fig. 3. Static eccentricity for an arbitrary $i$ th element is defined as in (3) and (4).

$$
\begin{aligned}
& e_{i, \mathrm{st}}=\sqrt{x_{i, \mathrm{st}}^{2}+y_{i, \mathrm{st}}^{2}} \\
& \theta_{i, \mathrm{st}}=\tan ^{-1}\left(\frac{y_{i, \mathrm{st}}}{x_{i, \mathrm{st}}}\right), \text { when }-\frac{\pi}{2}<\theta<\frac{\pi}{2}
\end{aligned}
$$

in which

$$
\begin{aligned}
x_{i, \mathrm{st}}= & e_{\mathrm{ini}, \mathrm{st}} \cos \theta_{\mathrm{ini}, \mathrm{st}} \\
& +\frac{2 i-1}{2 n}\left(e_{\mathrm{fin}, \mathrm{st}} \cos \theta_{\mathrm{fin}, \mathrm{st}}-e_{\mathrm{ini}, \mathrm{st}} \cos \theta_{\mathrm{ini}, \mathrm{st}}\right) \\
y_{i, \mathrm{st}}= & e_{\mathrm{ini}, \mathrm{st}} \sin \theta_{\mathrm{ini}, \mathrm{st}} \\
& +\frac{2 i-1}{2 n}\left(e_{\mathrm{fin}, \mathrm{st}} \sin \theta_{\mathrm{fin}, \mathrm{st}}-e_{\mathrm{ini}, \mathrm{st}} \sin \theta_{\mathrm{ini}, \mathrm{st}}\right)
\end{aligned}
$$

where $x_{i, \mathrm{st}}$ and $y_{i, \mathrm{st}}$ are the coordinates of the center of the $i$ th rotor element with respect to the $x_{1}-y_{1}$ coordinate system. The number of divided rotor elements is $n$, and therefore, $i=1,2$, $\ldots, n$. The static eccentricity of the initial side of the rotor is given by $e_{\mathrm{ini}, \mathrm{st}}$ and $\theta_{\mathrm{ini}, \mathrm{st}}$, whereas $e_{\mathrm{fin}, \mathrm{st}}$ and $\theta_{\mathrm{fin}, \mathrm{st}}$ present the static eccentricity of the final side of the rotor. Consequently, the axial-varying eccentricity condition is modeled by using divided elements that have individual eccentricity. Moreover, this model can be easily applied to the rotor simulation model using finite elements.

\section{Eccentricity by Motor Frame Vibration}

The UMP excites both the stator and the rotor. At the same time, the vibration of the motor frame changes the air gap

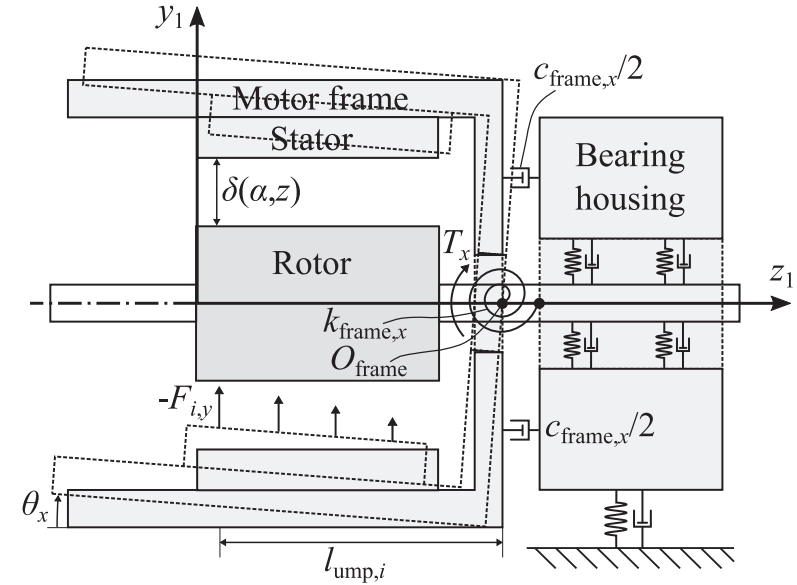

Fig. 4. Motor frame vibration model of the machine under study.

eccentricity. Therefore, it is necessary to predict the vibration behavior of the motor frame and consider its effect on the eccentricity. In this study, a simple model for the whole frame structure is developed to be easily applied to the air gap eccentricity calculation process. First, for the frame structure of the machine under study, a modal analysis was performed with ANSYS, and as a result, two frame-dominated modes were found [16]. Based on these mode shapes, it is assumed that the motor frame and the stator are rigid and considered one body, and the bearing housing is rigid and connected rigidly to the ground. This approach is taken as the focus of interest is on the effect of relative rotational vibration between the motor frame and the bearing housing. Based on these assumptions, the frame structure consisting of the motor frame and the bearing housing and excited by the UMP is modeled as a two-degree-of-freedom system as in Fig. 4. In this model, the motor frame is connected with the bearing housing by a rotational spring and a damper and rotated about a fixed origin $O_{\text {frame }}$. The developed model is limited to the machine structure of the study because the above assumptions are valid for this structure only. Detailed information about the machine structure is presented in Section V.

The rotational motion equation of the motor frame is formulated as

$$
\begin{aligned}
& \mathbf{I} \ddot{\boldsymbol{\theta}}+\mathbf{C} \dot{\boldsymbol{\theta}}+\mathbf{K} \boldsymbol{\theta}=\mathbf{T} \\
& \mathbf{I}=\left[\begin{array}{cc}
I_{\text {frame }, x} & 0 \\
0 & I_{\text {frame }, y}
\end{array}\right], \mathbf{C}=\left[\begin{array}{cc}
c_{\text {frame }, x} & 0 \\
0 & c_{\text {frame }, y}
\end{array}\right], \\
& \mathbf{K}=\left[\begin{array}{cc}
k_{\text {frame }, x} & 0 \\
0 & k_{\text {frame }, y}
\end{array}\right], \mathbf{T}=\left\{\begin{array}{l}
T_{x} \\
T_{y}
\end{array}\right\}, \\
& \boldsymbol{\theta}=\left\{\begin{array}{c}
\theta_{\text {stator }, x} \\
\theta_{\text {stator }, y}
\end{array}\right\}, \dot{\boldsymbol{\theta}}=\left\{\begin{array}{l}
\dot{\theta}_{\text {stator }, x} \\
\dot{\theta}_{\text {stator }, y}
\end{array}\right\}, \ddot{\boldsymbol{\theta}}=\left\{\begin{array}{l}
\ddot{\theta}_{\text {stator }, x} \\
\ddot{\theta}_{\text {stator }, y}
\end{array}\right\}
\end{aligned}
$$

where $\mathbf{I}$ is the mass moment of inertia of the motor frame and $\mathbf{C}$ and $\mathbf{K}$ are the rotational damping and stiffness of the motor frame, respectively. Rotational displacement, velocity, and acceleration vectors of the frame are denoted by $\boldsymbol{\theta}, \dot{\boldsymbol{\theta}}$, and $\ddot{\boldsymbol{\theta}}$. 
The moment caused by the $\operatorname{UMP}\left(F_{x}, F_{y}\right)$ acting on the frame can be calculated as

$$
\begin{aligned}
& T_{x}=-\sum_{i=1}^{n} F_{i, y} l_{\mathrm{ump}, i} \\
& T_{y}=-\sum_{i=1}^{n} F_{i, x} l_{\mathrm{ump}, i}
\end{aligned}
$$

When the motor frame is vibrated by the moment, the displacements of the stator bore at the $i$ th node where the UMP is applied are given by

$$
x_{\text {stator }, i}=l_{\text {ump }, i} \tan \theta_{y}, y_{\text {stator }, i}=l_{\text {ump }, i} \tan \theta_{x}
$$

where $l_{\mathrm{ump}, i}$ is the axial length between the stator rotation center and the node where the eccentricity is defined. Eccentricity generated by the displacement of the stator bore can be calculated by

$$
\begin{aligned}
& e_{\text {stator }, i}(t)=\sqrt{\left(x_{\text {stator }, i}(t)\right)^{2}+\left(y_{\text {stator }, i}(t)\right)^{2}} \\
& \theta_{\text {stator }, i}(t)=\tan ^{-1}\left(\frac{y_{\text {stator }, i}(t)}{x_{\text {stator }, i}(t)}\right)
\end{aligned}
$$

\section{Combined Eccentricity}

From previous eccentricity models, the combined eccentricity of the $i$ th element of an electrically active part of the rotor is defined as in (14) and (15).

$$
e_{\mathrm{comb}, i}(t)=\left\{\begin{array}{c}
\left(e_{i, \mathrm{st}} \cos \theta_{i, \mathrm{st}}+x_{2}(t)+x_{\mathrm{stator}, i}(t)\right)^{2} \\
+\left(e_{i, \mathrm{st}} \sin \theta_{i, \mathrm{st}}+y_{2}(t)+y_{\mathrm{stator}, i}(t)\right)^{2}
\end{array}\right\}^{1 / 2}
$$

$$
\theta_{\mathrm{comb}, i}(t)=\tan ^{-1}\left(\frac{e_{i, \mathrm{st}} \sin \theta_{i, \mathrm{st}}+y_{2}(t)+y_{\mathrm{stator}, i}(t)}{e_{i, \mathrm{st}} \cos \theta_{i, \mathrm{st}}+x_{2}(t)+x_{\mathrm{stator}, i}(t)}\right)
$$

\section{Analytical Calculation of the UMP by ECCENTRICITY AT PMSM}

In this section, an analytical UMP model for a permanent magnet synchronous motor is described. The basic concept is to determine the air gap flux by modulating the fundamental component of the magnetomotive force (MMF) wave considering the air gap permeance and calculate the corresponding force components by the Maxwell stress tensor method.

In the model, the fundamental component of the air gap MMF is taken from the study by Chen et al. [11] using the same assumptions but with a slight modification, i.e., also the relative permeability of the permanent magnet is considered to calculate the rotor MMF as in (17). The amplitude of the fundamental MMF of the air gap is written as

$$
\begin{gathered}
F_{\mathrm{m}}=\sqrt{F_{\mathrm{sm}}^{2}+F_{\mathrm{rm}}^{2}-2 F_{\mathrm{sm}} F_{\mathrm{rm}} \sin \varphi} \\
F_{\mathrm{rm}}=\frac{4 B_{\mathrm{r}} h_{\mathrm{m}}}{\pi \mu_{\mathrm{r}, \mathrm{PM}} \mu_{0}} \sin \left(\frac{\alpha_{\mathrm{p}} \pi}{2}\right), F_{\mathrm{sm}}=\frac{\sqrt{2} m N k_{\mathrm{w}}}{\pi p} I
\end{gathered}
$$

TABLE I

PARAMETERS OF THE ELECTRICAL MACHINE UNDER STUDY

\begin{tabular}{lll}
\hline \hline Symbol & \multicolumn{1}{c}{ QUANTITY } & \multicolumn{1}{c}{ Value } \\
\hline$P$ & rated power & $30 \mathrm{~kW}$ \\
$n$ & rated speed & $4100 \mathrm{rpm}$ \\
$T$ & rated torque & $69.9 \mathrm{~N} \cdot \mathrm{m}$ \\
$I$ & rated current & $41 \mathrm{~A}$ \\
$k_{\mathrm{W}}$ & winding factor & 0.93 \\
$p$ & number of pole pairs & 3 \\
$m$ & number of phases & 3 \\
$N$ & number of series turns per phase & 48 \\
$B_{\mathrm{r}}$ & magnetic remanence & $1.07 \mathrm{~T}$ \\
$h_{\mathrm{m}}$ & thickness of permanent magnet & $3.5 \mathrm{~mm}$ \\
$\mu_{0}$ & air permeability & $4 \pi \times 10^{7} \mathrm{~N} / \mathrm{A}^{2}$ \\
$\mu_{\mathrm{r}, \mathrm{PM}}$ & relative permeability of permanent magnet & 1.0445 \\
$\alpha_{\mathrm{p}}$ & magnetic pitch/pole ratio & 0.75 \\
$\delta_{0}$ & mean air gap length including rotor surface & $5.5 \mathrm{~mm}$ \\
& thickness (3 mm) & \\
$R_{\mathrm{r}}$ & outer radius of permanent magnet rotor & $77 \mathrm{~mm}$ \\
$l$ & length of permanent magnet rotor & $126 \mathrm{~mm}$ \\
$\varphi$ & power factor angle & 0.142 \\
$I_{\text {frame }}$ & mass moment of inertia of the frame & $4 \mathrm{~kg} \cdot \mathrm{m}^{2}$ \\
$k_{\text {frame }, x}$ & rotation stiffness of the frame about $x$-axis & $1.20 \cdot 10^{6} \mathrm{~N} / \mathrm{m}$ \\
$k_{\text {frame, } y}$ & rotation stiffness of the frame about $y$-axis & $7.57 \cdot 10^{5} \mathrm{~N} / \mathrm{m}$ \\
$c_{\text {frame }, x}$ & rotation damping of the frame about $x$-axis & $81.81 \mathrm{~N} \cdot \mathrm{s} / \mathrm{m}$ \\
$c_{\text {frame, } y}$ & rotation damping of the frame about $y$-axis & $65.09 \mathrm{~N} \cdot \mathrm{s} / \mathrm{m}$ \\
\hline \hline
\end{tabular}

where $F_{\mathrm{sm}}$ and $F_{\mathrm{rm}}$ are the amplitudes of the fundamental MMF waves for the stator and the permanent magnet rotor, respectively. The variables are explained in Table I. integration using the Maxwell stress over the rotor surface as ary and $\alpha$ is a variable for defining air-gap's circumferential location on the rotor surface as in Fig. 1. lished using two modeling approaches. In the first approach, the UMP is applied to the rotor model as a linear spring element. In the second approach, the UMP is applied as a nonlinear force. stiffness is first defined employing the UMP model developed by Guo et al. [9] with several assumptions. In the UMP model, if oscillating terms except the first constant term are ignored and the power series having only the first two terms as in (20) are used under the assumption $\varepsilon^{2} \ll 1$, the UMP model can be simplified as in (21) and (22).
The resulting force of the UMP can be obtained by direct

$$
\begin{aligned}
& F_{x}=\int_{0}^{2 \pi} \sigma R_{\mathrm{r}} l \cos \alpha d \alpha \\
& F_{y}=\int_{0}^{2 \pi} \sigma R_{\mathrm{r}} l \sin \alpha d \alpha
\end{aligned}
$$

Here, $\sigma$ is the Maxwell stress normal to the iron and air bound-

\section{Modeling of A Rotor-Bearing System With UMP}

In this section, the rotor-bearing model with UMP is estab-

For the simulation using the first approach, a linearized UMP

$$
\begin{gathered}
\frac{1}{1-\varepsilon^{2}} \approx 1+\varepsilon^{2}, \sqrt{1-\varepsilon^{2}} \approx 1-\frac{\varepsilon^{2}}{2} \\
F_{x}(\varepsilon, \theta)=\frac{R l \pi \mu_{0}}{2 \delta_{0}^{2}} F_{\mathrm{m}}^{2}\left(\varepsilon+\frac{5}{4} \varepsilon^{3}+\frac{5}{16} \varepsilon^{5}+\frac{1}{16} \varepsilon^{7}\right) \cos \theta
\end{gathered}
$$




$$
F_{y}(\varepsilon, \theta)=\frac{R l \pi \mu_{0}}{2 \delta_{0}^{2}} F_{\mathrm{m}}^{2}\left(\varepsilon+\frac{5}{4} \varepsilon^{3}+\frac{5}{16} \varepsilon^{5}+\frac{1}{16} \varepsilon^{7}\right) \sin \theta
$$

Moreover, if it is assumed that the eccentricity has purely horizontal or vertical direction components, i.e., the coupled terms between the $x$ and $y$ directions are ignored, the UMP stiffness ( $\left.k_{\mathrm{ump}, x}, k_{\mathrm{ump}, y}\right)$ can be obtained as follows. When $\theta$ is zero (pure $x$-direction eccentricity, $x=\delta_{0} \varepsilon$ ),

$$
F_{x}=\frac{R l \pi \mu_{0}}{2 \delta_{0}^{3}} F_{\mathrm{m}}^{2}\left(x+\frac{5 x^{3}}{4 \delta_{0}^{2}}+\frac{5 x^{5}}{16 \delta_{0}^{4}}+\frac{x^{7}}{16 \delta_{0}^{6}}\right)
$$

When $\theta$ is $\pi / 2$ (pure $y$-direction eccentricity, $y=\delta_{0} \varepsilon$ ),

$$
F_{y}=\frac{R l \pi \mu_{0}}{2 \delta_{0}^{3}} F_{\mathrm{m}}^{2}\left(y+\frac{5 y^{3}}{4 \delta_{0}^{2}}+\frac{5 y^{5}}{16 \delta_{0}^{4}}+\frac{y^{7}}{16 \delta_{0}^{6}}\right)
$$

Above, the UMP force is linearized as a derivative in a static eccentricity $\left(x_{\mathrm{st}}, y_{\mathrm{st}}\right)$, and the UMP stiffness is presented as

$$
\left.k_{\mathrm{ump}, x} \approx \frac{d F_{x}(x)}{d x}\right|_{x_{\mathrm{st}}},\left.k_{\mathrm{ump}, y} \approx \frac{d F_{y}(y)}{d y}\right|_{y_{\mathrm{st}}}
$$

In conclusion, the equation of motion with UMP can be established by using the UMP stiffness vector $\mathbf{K}_{\text {ump }}$ linearized at a given static eccentricity as

$$
\mathbf{M} \ddot{\mathbf{q}}+(\mathbf{C}+\Omega \mathbf{G})\left(\dot{\mathbf{q}}+\left(\mathbf{K}-\mathbf{K}_{\mathrm{ump}}\right) \mathbf{q}=\mathbf{F}_{\mathrm{ub}}+\mathbf{F}_{\mathrm{g}}\right.
$$

where $\mathbf{q}$ is the displacement vector and $\mathbf{M}, \mathbf{C}, \mathbf{G}$, and $\mathbf{K}$ are the mass, damping, gyroscopic, and stiffness matrices, respectively. Term $\Omega$ is the rotor angular velocity. Correspondingly, $\mathbf{F}_{\mathrm{ub}}$ and $\mathbf{F}_{\mathrm{g}}$ denote the unbalance force and the gravity force, respectively.

In the second method, UMP is added as an external force $\left(\mathbf{F}_{\text {ump }}\right)$ directly to the equation of motion as follows

$$
\mathbf{M} \ddot{\mathbf{q}}+(\mathbf{C}+\Omega \mathbf{G}) \dot{\mathbf{q}}+\mathbf{K q}=\mathbf{F}_{\mathrm{ub}}+\mathbf{F}_{\mathrm{g}}+\mathbf{F}_{\mathrm{ump}}
$$

\section{Simulation}

In this section, simulations using the rotor-bearing system models in Section IV are presented. The simulations were conducted for a centrifugal pump with an integrated PMSM. Results for several cases were obtained and compared to identify the effects of UMP on the vibration of the machine.

\section{A. Electrical Machine Under Study}

The machine under study consists of a motor, a bearing unit, and a volute case. It has an overhang structure in which the rotor is not supported on the motor rear side; further, the impeller is not considered. The structure of the machine is shown in Fig. 5, and the machine parameters are given in Table I. The mass unbalance of the rotor is located at the face of the nonpump side, and its magnitude is $110.25 \mathrm{~g} \cdot \mathrm{mm}$ corresponding to the balancing grade $\mathrm{G} 2.5$. Two angular contact ball bearings support the rotor, and their stiffness is estimated by using a simple method proposed by Gargiulo [17]. According to this method, the stiffness is calculated by considering the ball diameter, the ball numbers, and the bearing radial load. Here, the bearing load is estimated on the assumption of $20 \%$ static eccentricity and a

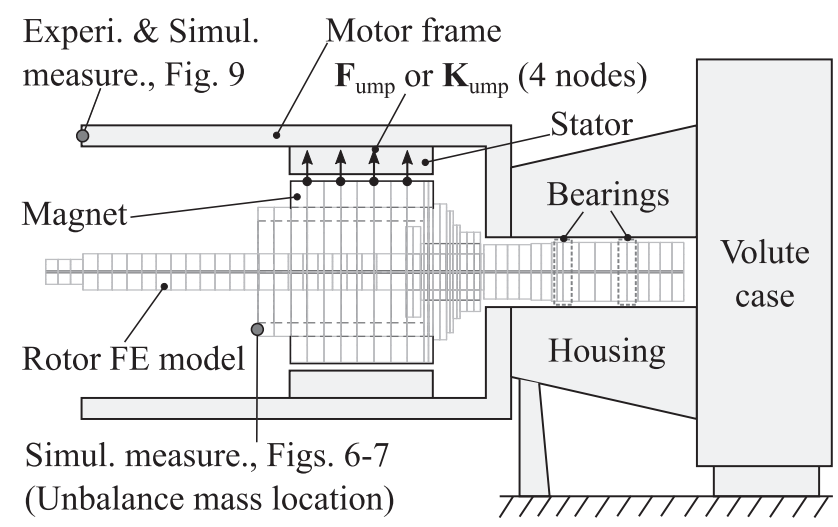

Fig. 5. Structure of the electrical machine under study.

$20 \mu \mathrm{m}$ (0-peak) rotor whirling vibration condition, because the result of the $20 \%$ eccentricity case will be compared with the experimental result. In conclusion, each bearing has constant stiffness $\left(k_{\text {bearing }}=1.4 \cdot 10^{8} \mathrm{~N} / \mathrm{m}\right)$ and damping $\left(3.5 \cdot 10^{3} \mathrm{~N} \cdot \mathrm{s} / \mathrm{m}\right)$ in both the horizontal and vertical directions. Here, damping is estimated as $2.5 \cdot 10^{-5} \cdot k_{\text {bearing }}$ based on the suggestion presented in [18].

\section{B. Simulation Method}

For the rotordynamic analysis, the rotor is modeled with beam finite elements that have four degrees of freedom per node. This model assumes that there is no displacement in the axial direction and no rotation around the rotor axis. To consider axial-varying static eccentricity, the electrically active part of the rotor is divided into four parts and individual static eccentricity is applied to each part. In this study, seven cases in total are simulated; without UMP, with UMP in three conditions of eccentricity (mixed and axial-varying eccentricity with $0-0 \%, 10-0 \%$ and $20-0 \%$ static eccentricities), and three cases with UMP including frame vibration. The axial-varying static eccentricity is expressed as initial side eccentricity and final side eccentricity. The direction angle of the static eccentricity is set to zero in all cases. The static eccentricities of the divided rotor parts are calculated using (3) to (6).

For the simulation using the linear spring model of the UMP, the UMP stiffness is calculated using the process presented in Section IV. To consider axial-varying static eccentricity, the individual UMP stiffness is calculated at the obtained static eccentricity of each rotor part and applied to the node of the rotor part. Total UMP stiffness values in the horizontal direction for the entire rotor are calculated as $1.399 \cdot 10^{6}$ for $0-0 \%, 1.403 \cdot 10^{6}$ for $10-0 \%$, and $1.414 \cdot 10^{6} \mathrm{~N} / \mathrm{m}$ for $20-0 \%$ static eccentricity. The vertical direction stiffness is the same $1.399 \cdot 10^{6} \mathrm{~N} / \mathrm{m}$ for all cases because there is no eccentricity in the vertical direction. The motion equation is defined as an eigenvalue problem and the critical speeds are calculated by solving this problem. However, the motor frame vibration model cannot be included in this method.

In the simulation applying the external force model of the UMP, a time transient analysis using numerical integration is 


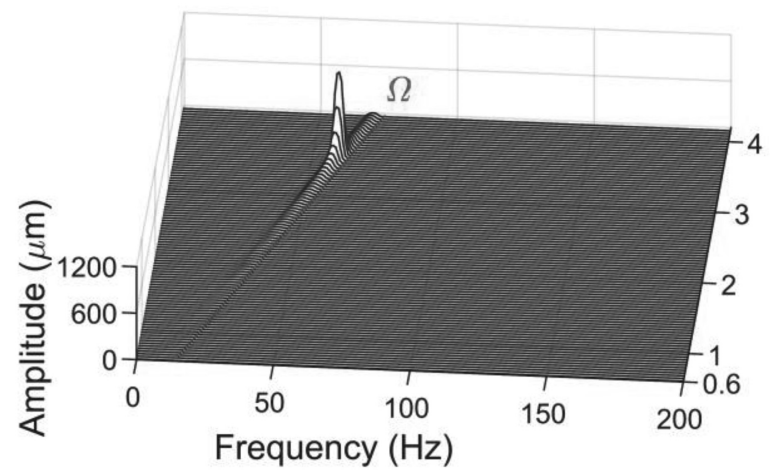

(a) Without UMP

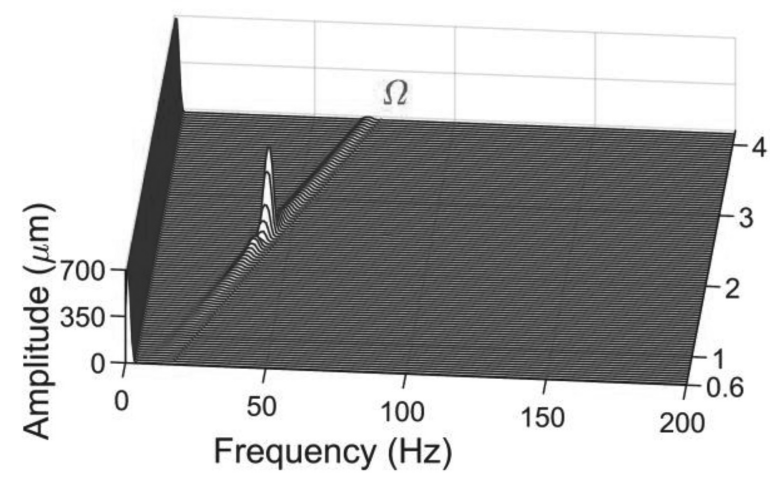

(c) With UMP (mixed eccentricity with $20-0 \%$ static eccentricity)

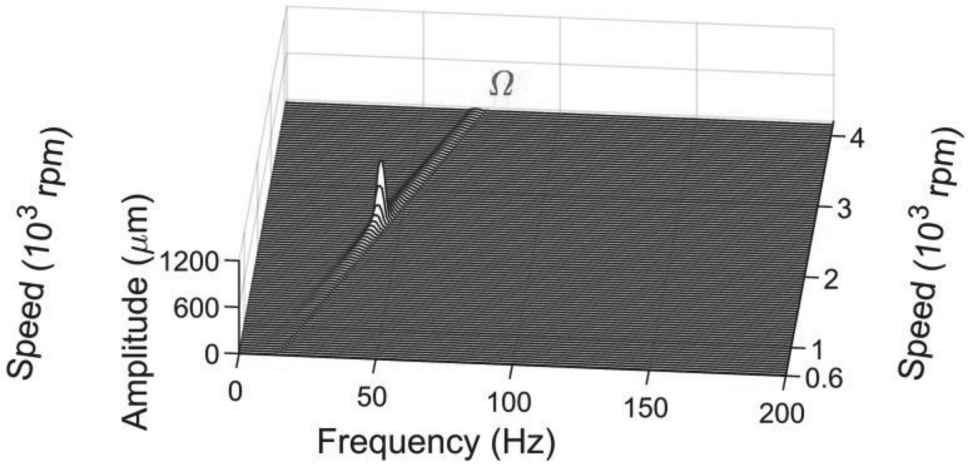

(b) With UMP (only dynamic eccentricity)

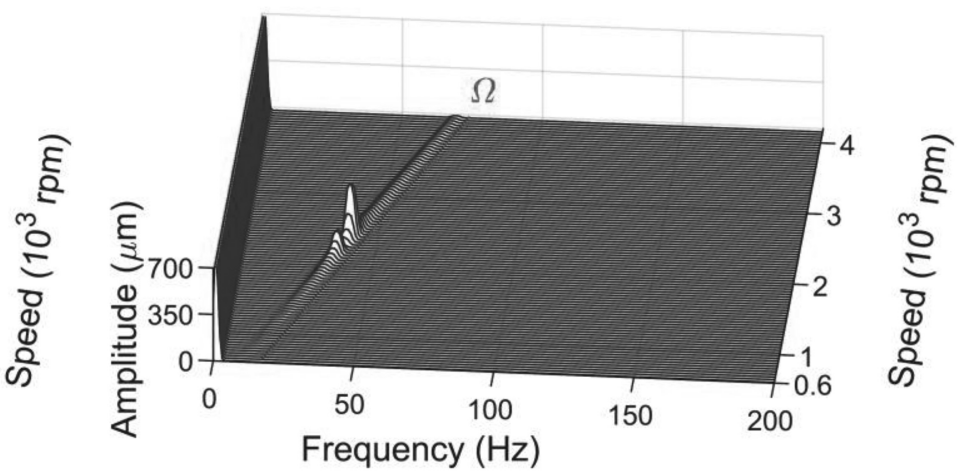

(d) With UMP \& frame model (mixed eccentricity with $20-0 \%$ static eccentricity)

Fig. 6. 3D spectral map of horizontal direction displacement $(\mu \mathrm{m})$ at the unbalance mass location to determine a change in critical speeds.

conducted to solve the motion equation. The numerical integration is performed using the ode $15 \mathrm{~s}$ function in MATLAB. In the analysis process, the rotor location is updated at every time step and the UMP is calculated using eccentricity from the updated rotor location. For the simulation including frame vibration, the inertia, stiffness, and damping of the frame presented in Table I are used. These values were predicted by using two natural frequencies $(74$ and $93 \mathrm{~Hz})$ related to the frame's rotational vibration modes determined by experimental measurement [16]. To investigate the UMP effects, a ramp from 10 to $70 \mathrm{~Hz}$ in $60 \mathrm{~s}$ is simulated for all cases.

\section{Simulation Results}

From the simulation using the linear spring model, critical speeds of the rotor are obtained for four cases without frame vibration. Again, $3 \mathrm{D}$ spectral maps of the rotor vibration were obtained from the simulation using the external force model. Here, displacement vibration is measured at the rotor node where the unbalance mass is located as in Fig. 5., because rotor vibration can be only measured when not including the frame model. For a comparison with the experimental results, in the case including the frame model, also velocity is measured at the same frame location with the experimental measurement, which is presented in Section IV on experimental verification.
The $3 \mathrm{D}$ spectral maps for the four cases: without UMP, with UMP at $0-0 \%$ and $0-20 \%$ axial-varying static eccentricity, and with UMP including frame vibration at $20-0 \%$ axial-varying static eccentricity are presented in Figs. 6 and 7. From the peak point of the 3D spectral map, the first backward/forward critical speeds can be found. Changes in the critical speeds caused by the UMP are detected and presented in Table II. The negative stiffness effect was observed similarly in both simulation methods. The critical speeds were decreased by the UMP, and this effect was slightly amplified by frame vibration. The effect of static eccentricity on the negative stiffness was small in comparison with the effect of dynamic eccentricity. This finding is in agreement with the calculation result indicating that the UMP stiffness change from the static eccentricity is small.

Moreover, the effect of static eccentricity on the negative stiffness was shown with only a little difference between two methods. In the method using the linear spring model of the UMP, the effect by static eccentricity appeared to be smaller than in the other method. Furthermore, it is shown that the excitation of the backward whirling mode increases when the static eccentricity increases as shown in Fig. 6. The backward whirling mode is excited by the anisotropic support stiffness [19], which is amplified by the anisotropic negative stiffness caused by static eccentricity.

To determine the additional excitations from the UMP, 3D spectral maps with a decreased range of the $\mathrm{z}$ axis are presented 


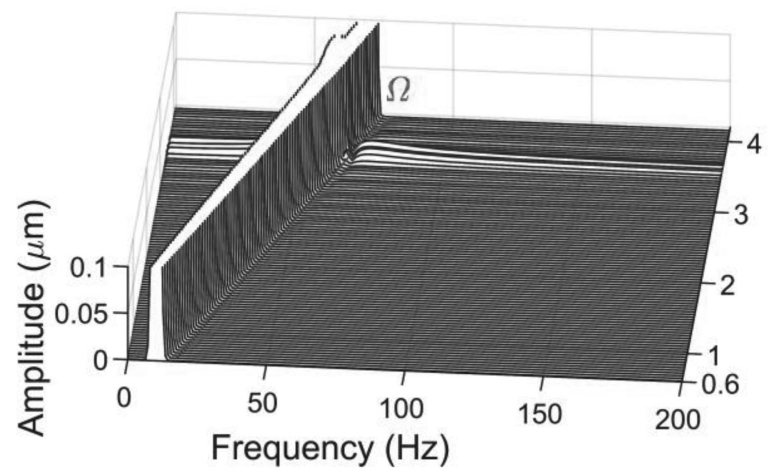

(a) Without UMP

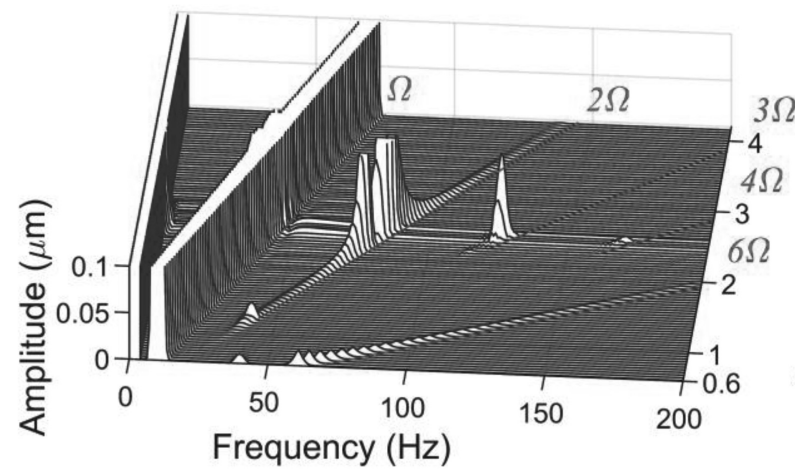

(c) With UMP (mixed eccentricity with $20-0 \%$ static eccentricity)

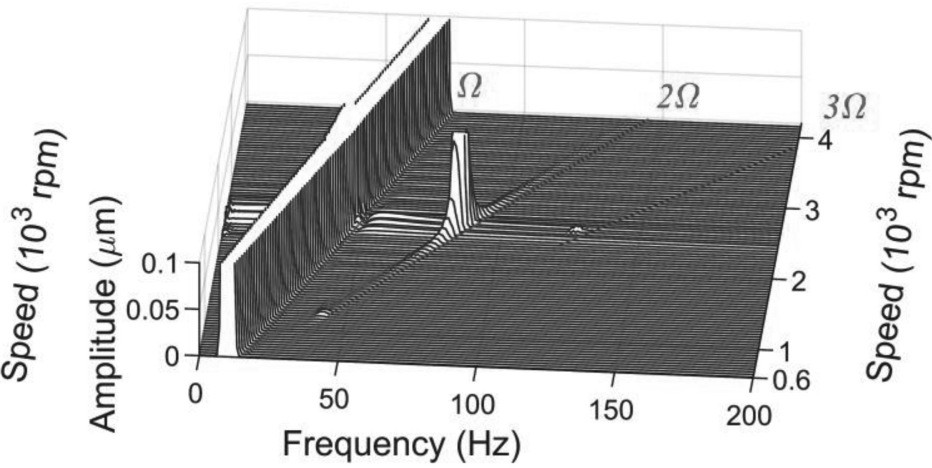

(b) With UMP (only dynamic eccentricity)

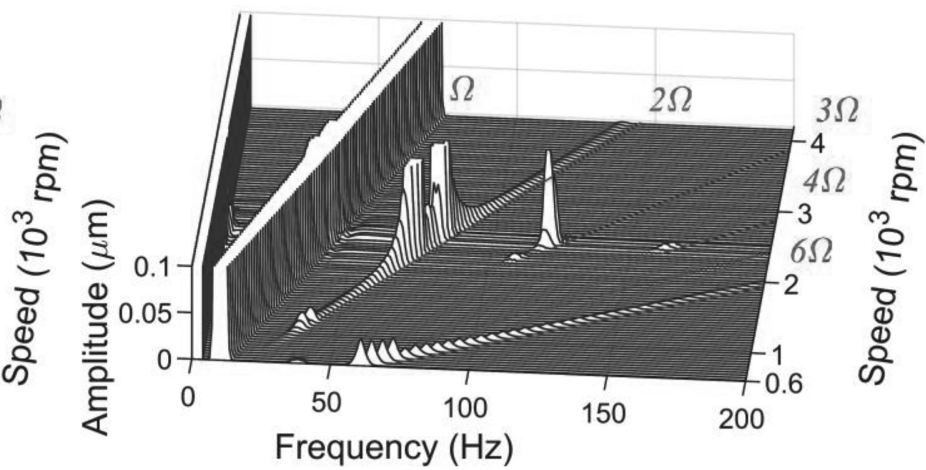

(d) With UMP \& frame model (mixed eccentricity with $20-0 \%$ static eccentricity)

Fig. 7. 3D spectral map of horizontal direction displacement $(\mu \mathrm{m})$ at unbalance mass location to determine higher frequency excitations.

TABLE II

Change in CRitical Speeds (Simulation 1: Linear SpRing Model, Simulation 2: NONLINEAR ForCE MODEL)

\begin{tabular}{|c|c|c|c|}
\hline Method & CASE & $\begin{array}{c}\text { 1ST BW } \\
\text { CRITICAL } \\
\text { SPEED, Hz }\end{array}$ & $\begin{array}{c}\text { 1ST FW } \\
\text { CRITICAL } \\
\text { SPEED, HZ }\end{array}$ \\
\hline \multirow{4}{*}{$\begin{array}{l}\text { Simulation1 } \\
\text { (Eigenvalue) }\end{array}$} & Without UMP & 54.1 & 59.1 \\
\hline & With UMP $(0-0 \%)$ & $38.6(\downarrow 15.5)$ & $42.2(\downarrow 16.9)$ \\
\hline & With UMP $(10-0 \%)$ & $38.6(\downarrow 15.5)$ & $42.1(\downarrow 17.0)$ \\
\hline & With UMP $(20-0 \%)$ & $38.5(\downarrow 15.6)$ & $42.0(\downarrow 17.1)$ \\
\hline \multirow{7}{*}{$\begin{array}{l}\text { Simulation2 } \\
\text { (Numerical } \\
\text { time } \\
\text { integration) }\end{array}$} & Without UMP & $-^{*}$ & 59.7 \\
\hline & With UMP $(0-0 \%)$ & $42.2(\downarrow 11.9)$ & $43.8(\downarrow 15.9)$ \\
\hline & With UMP $(10-0 \%)$ & $38.2(\downarrow 15.9)$ & $42.2(\downarrow 17.5)$ \\
\hline & With UMP $(20-0 \%)$ & $37.4(\downarrow 16.7)$ & $41.4(\downarrow 18.3)$ \\
\hline & $\begin{array}{l}\text { With UMP }(0-0 \%) \\
\& \text { frame vibration }\end{array}$ & $37.4(\downarrow 16.7)$ & $41.4(\downarrow 18.3)$ \\
\hline & $\begin{array}{l}\text { With UMP }(10-0 \%) \\
\& \text { frame vibration }\end{array}$ & $37.4(\downarrow 16.7)$ & $41.4(\downarrow 18.3)$ \\
\hline & $\begin{array}{l}\text { With UMP }(20-0 \%) \\
\& \text { frame vibration }\end{array}$ & $36.6(\downarrow 17.5)$ & $39.8(\downarrow 19.9)$ \\
\hline
\end{tabular}

${ }^{*}$ Backward mode (without UMP case) is not excited in Simulation 2, and therefore, the effect of UMP is found by comparing with the critical speed $(54.1 \mathrm{~Hz})$ in Simulation 1. in Fig. 7. It is found that harmonics with $2 \Omega, 3 \Omega, 4 \Omega$, $5 \Omega$, and $6 \Omega$ (2.line frequency) frequencies are generated, and their amplitudes increase when the static eccentricity increases. In particular, it is shown that the $6 \Omega$ frequency component occurs especially when static eccentricity is present. Finally,
TABLE III

VibRATION FREQUENCIES IN SiMULATION RESUlT With UMP AS AN EXTERNAL NONLINEAR FORCE

\begin{tabular}{ll}
\hline \hline \multicolumn{1}{c}{ CASE } & \multicolumn{1}{c}{ MAIN OCCURRENCE FREQ. } \\
\hline Without UMP & $\Omega$ \\
With UMP (0-0\% static) & $\Omega, 2 \Omega$ \\
With UMP (10-0, 20-0\% static) & $\Omega, 2 \Omega, 6 \Omega\left(2 \cdot\right.$ line freq. $\left.{ }^{*}\right)$ \\
\hline \hline
\end{tabular}

${ }^{*}$ Line freq. $=p \Omega=3 \Omega, p$ : pole pair no.
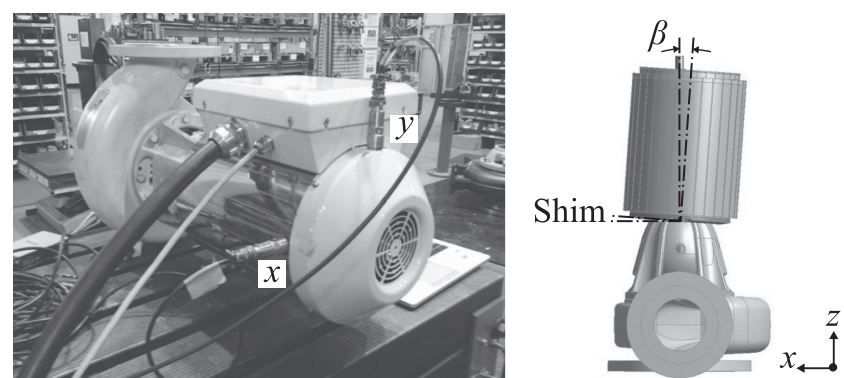

Fig. 8. Pump test rig, (left) two accelerometers for measurement, (right) axialvarying static eccentricity by shim.

it is observed that the UMP effect is slightly amplified by frame vibration. The critical speeds are lower, and especially the excitations that meet the frame natural frequency are significantly amplified. 


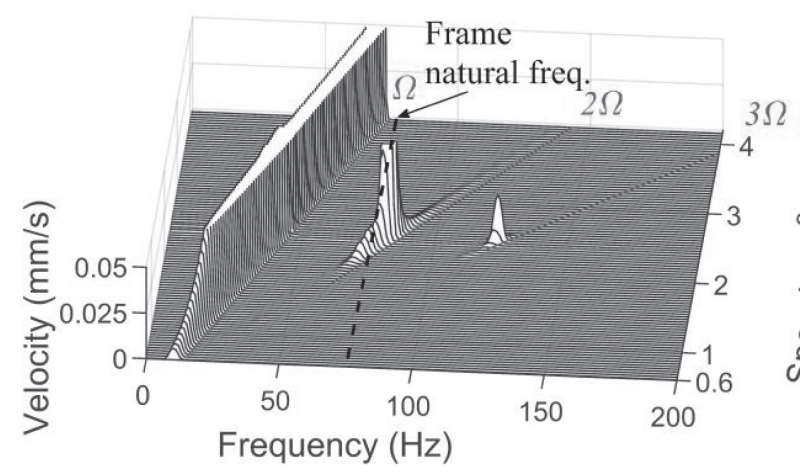

(a) Simulation, $0-0 \%$ axial-varying static eccentricity

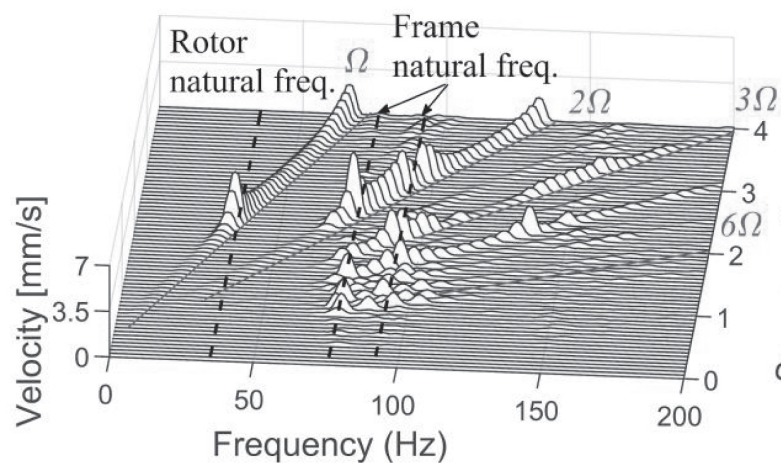

(c) Experiment, $0-0 \%$ axial-varying static eccentricity

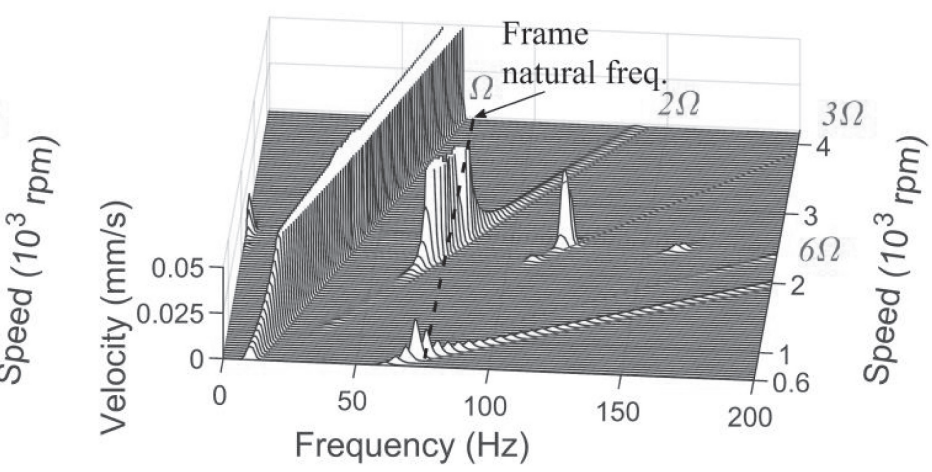

(b) Simulation, $20-0 \%$ axial-varying static eccentricity

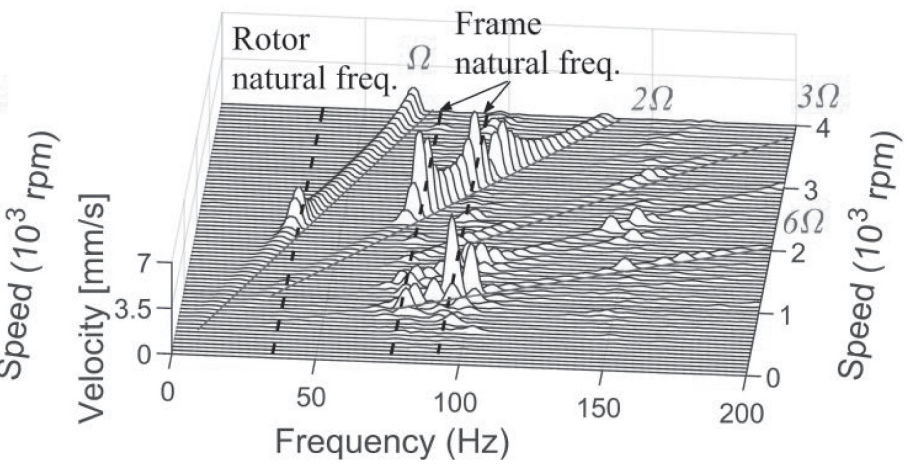

(d) Experiment, 20-0\% axial-varying static eccentricity

Fig. 9. Comparison between the 3D spectral maps for the simulation and the experimental results of horizontal direction velocity ( $\mathrm{mm} / \mathrm{s})(\Omega$ : rotating speed).

\section{EXPERIMENTAL VERIFICATION}

To verify the simulation results, experiments were carried out with a pump test rig. Axial-varying static eccentricity was adjusted by placing a shim between the bearing housing and the motor frame as in Fig. 8. The eccentricity was checked with a customized feeler gauge in which the measurement limits were set at $0.25 \mathrm{~mm}$ increments. For the vibration measurement, two accelerometers (IMI VO-622) were attached to the nonpump side of the motor frame in the $x$ and $y$-directions as in Fig. 8 .

A ramp from 0 to $4100 \mathrm{rpm}$ in $70 \mathrm{~s}$ was driven and 3D spectral maps for the velocity vibration of the frame were obtained as a result. In this experiment, only the cases including UMP were investigated because it is impossible to drive the rotor without the effect of the UMP. Therefore, higher frequency excitations from the UMP and the effect caused by the variation of the static eccentricity can be seen from the experimental results, but the negative stiffness effect from the presence of the UMP cannot be accurately verified. 3D spectral maps for vibration in horizontal direction for two cases with $0-0 \%$ and $20-0 \%$ axial-varying static eccentricities are presented in Fig. 9 with simulation results measured at the same location using the same vibration unit.

The experimental results show that harmonic excitations occur similarly to the simulation result, and these components are amplified at frame natural frequencies. As mentioned above in the frame vibration modeling section, the whole frame has two natural frequencies ( 73 and $93 \mathrm{~Hz}$ ) within the operating speed range. However, in simulation results presented in Fig. 9, the amplification is shown at only first natural frequency, of which the mode shape is related with vibration in horizontal direction. Moreover, it is shown that the $2 \Omega$ and $6 \Omega$ frequency components are significantly amplified by the static eccentricity. These results are in agreement with the simulation results. However, the amplitudes of these components are much higher than the ones found in the simulation results. This discrepancy is probably due to the simplifications made in the modeling of frame vibration; the topic, however, requires further study.

\section{CONCLUSION}

An eccentricity model including mixed eccentricity and axialvarying eccentricity considering frame vibration was developed. Based on the eccentricity model, analyical unbalanced magnetic pull (UMP) model was applied to the rotor simulation model of a centrifugal pump with a PMSM. From simulation results for several cases, the vibration effects caused by the UMP were studied, and the results were verified by an experimental analysis of a pump test rig. 
The key findings of the study on the effect of UMP on vibration and conclusions about the simulations can be summarized as follows.

1) The negative stiffness effect caused by the UMP decreases rotor critical speeds. This effect is mainly due to dynamic eccentricity and slightly amplified by static eccentricity and frame vibration. Moreover, the anisotropy of static eccentricity amplifies the excitation of the backward whirling mode.

2) Additional vibration components with frequencies of $2 \Omega$, $6 \Omega$ (2.line frequency), and other speed multiple frequencies are generated by the UMP. The vibration component of the 2 -line frequency is mainly generated when static eccentricity is present, and its amplitude is increased when the static eccentricity increases.

3) The simulation method using a linear negative spring model of UMP can be used to estimate the effect of negative stiffness on rotor critical speeds. However, the method applying UMP as an external force can only be used for prediction of higher frequency excitations produced by UMP.

4) A comparison between the simulations and the experimental results showed that the proposed simulation model can be used to predict electromagnetic excitations caused by UMP from air gap eccentricity.

5) In the experimental results, the amplitude of the electromechanical excitation at the natural frequency of the frame was significantly higher than in the simulations. This difference is probably due to the assumptions used in the modeling of the frame vibration. Hence, further studies on the modeling of the frame vibration effects are required.

\section{REFERENCES}

[1] A. J. Pina Ortega and L. Xu, "Investigation of effects of asymmetries on the performance of permanent magnet synchronous machines," IEEE Trans. Energy Convers., vol. 32, no. 3, pp. 1002-1011, Sep. 2017.

[2] Z. Q. Zhu, D. Ishak, D. Howe, and J. Chen, "Unbalanced magnetic forces in permanent-magnet brushless machines with diametrically asymmetric phase windings," IEEE Trans. Ind. Appl., vol. 43, no. 6, pp. 1544-1553, Nov./Dec. 2007.

[3] Z. J. Liu and J. T. Li, "Accurate prediction of magnetic field and magnetic forces in permanent magnet motors using an analytical solution," IEEE Trans. Energy Convers., vol. 23, no. 3, pp. 717-726, Sep. 2008.

[4] M. Donát, "Computational modelling of the unbalanced magnetic pull by finite element method," Procedia Eng., vol. 48, pp. 83-89, 2012.

[5] D. G. Dorrell, W. T. Thomson, and S. Roach, "Analysis of airgap flux, current, and vibration signals as a function of the combination of static and dynamic airgap eccentricity in 3-phase induction motors," IEEE Trans. Ind. Appl., vol. 33, no. 1, pp. 24-34, Jan./Feb. 1997.

[6] D. G. Dorrell, "Sources and characteristics of unbalanced magnetic pull in three-phase cage induction motors with axial-varying rotor eccentricity," IEEE Trans. Ind. Appl., vol. 47, no. 1, pp. 12-24, Jan./Feb. 2011.

[7] Y. Li and Z. Q. Zhu, "Cogging torque and unbalanced magnetic force prediction in PM machines with axial-varying eccentricity by superposition method," IEEE Trans. Magn., vol. 53, no. 11, Nov. 2017, Art. no. 1400404.

[8] A. Tenhunen, T. Benedetti, T. P. Holopainen, and A. Arkkio, "Electromagnetic forces of the cage rotor in conical whirling motion," IEE Proc.Electr. Power Appl., vol. 150, no. 5, pp. 563-568, Sep. 2003.

[9] D. Guo, F. Chu, and D. Chen, "The unbalanced magnetic pull and its effects on vibration in a three-phase generator with eccentric rotor," J. Sound Vib., vol. 254, no. 2, pp. 297-312, 2003.

[10] C. Di, X. Bao, H. Wang, Q. Lv, and Y. He, "Modeling and analysis of unbalanced magnetic pull in cage induction motors with curved dynamic eccentricity," IEEE Trans. Magn., vol. 51, no. 8, p. 8106507, Aug. 2015.
[11] X. Chen, S. Yuan, and Z. Peng, "Nonlinear vibration for PMSM used in HEV considering mechanical and magnetic coupling effects," Nonlinear Dyn., vol. 80, no. 1-2, pp. 541-552, 2015.

[12] C. Xiang, F. Liu, H. Liu, L. Han, and X. Zhang, "Nonlinear dynamic behaviors of permanent magnet synchronous motors in electric vehicles caused by unbalanced magnetic pull," J. Sound Vib., vol. 371, pp. 277-294, 2016.

[13] P. Lošák and R. Vlach, "Study of the response of the shaft loaded by unbalanced magnetic pull," in Proc. 16th Int. Conf. Mechatronics, Mechatronika, 2014, pp. 79-84.

[14] X. Xu, Q. Han, and F. Chu, "Nonlinear vibration of a generator rotor with unbalanced magnetic pull considering both dynamic and static eccentricities," Arch. Appl. Mech., vol. 86, no. 8, pp. 1521-1536, 2016.

[15] P. Pennacchi, "Computational model for calculating the dynamical behaviour of generators caused by unbalanced magnetic pull and experimental validation," J. Sound Vib., vol. 312, no. 1-2, pp. 332-353, 2008.

[16] A. Posa, "Vibration behavior of a centrifugal pump with integrated permanent magnet motor," M.S. thesis, Dept. Mech. Eng., Lappeenranta Univ. Technol., Lappeenranta, Finland, 2016.

[17] E. P. J. Gargiulo, "A simple way to estimate bearing stiffness," Mach. Des., vol. 52, no. 17, pp. 107-110, 1980.

[18] E. Krämer, Dynamics of Rotors and Foundations. Berlin Heidelberg, Germany: Springer-Verlag GmbH, 1993.

[19] L. M. Greenhill and G. A. Cornejo, "Critical speeds resulting from unbalance excitation of backward whirl modes," in Proc. Des. Eng. Tech. Conf., vol. 84-2, 1995, pp. 991-1000.

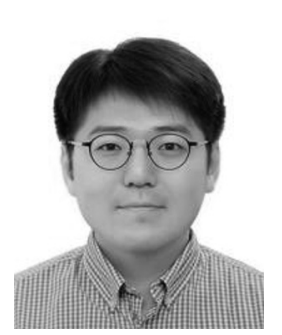

Heesoo Kim was born in Seoul, Korea, in 1979. He received the B.S. and M.S. degrees in mechanical engineering from Hanyang University, Seoul, Korea, in 2005 and 2007, respectively. After graduation, he worked as a Turbocharger Development Engineer for marine and vehicle engines. From 2017, he is working toward the Ph.D. degree at the Department of Mechanical Engineering, LUT University, Lappeenranta, Finland. His research interests include rotordynamics for electrical machines, specifically study of electromechanical interaction from air gap eccentricity, stator deformation, and other geometric non-idealities.

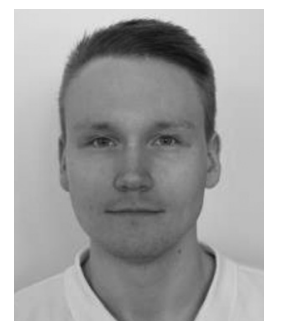

Atte Posa received the M.Sc. degree in mechanical engineering from LUT University, Finland, in 2016. After the master's studies, he started working as Product Development Engineer in Sulzer Pumps Finland Oy, Kotka, Finland. His work as Product Development Engineer is focused on mechanical seals and sealing systems.

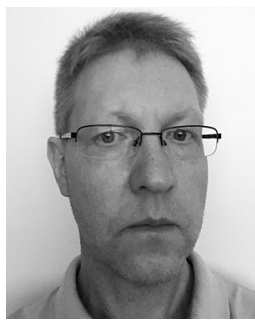

Janne Nerg (M'99-SM'12) received the M.Sc degree in electrical engineering, the Licentiate of Science (Technology) degree, and the D. Sc. (Technology) degree from LUT University, Lappeenranta, Finland, in 1996, 1998, and 2000, respectively. He is currently an Associate Professor with the Department of Electrical Engineering at LUT University. His research interests include electrical machines and drives, especially electromagnetic and thermal modeling and design of electromagnetic devices.

\section{4} 95

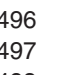

8

03

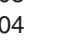

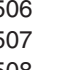

9

10

511
512
513

14

15

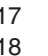
19

0

.




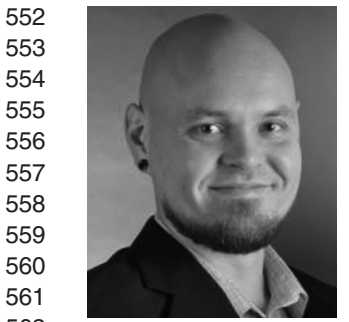

Janne Heikkinen received the M.Sc. degree in mechanical engineering from LUT University, Lappeenranta, Finland, in 2010. After the master's studies, he started his research career as a Ph.D. student at LUT University. He successfully defended his Doctoral Dissertation and received the D.Sc. degree from LUT University in 2014. He is currently working as a Postdoctoral Researcher in Laboratory of Machine Dynamics at LUT University. His research interests include rotating electric machines, especially highspeed machinery. His main expertise is in rotordynamics, structural vibrations, and vibration measurements.

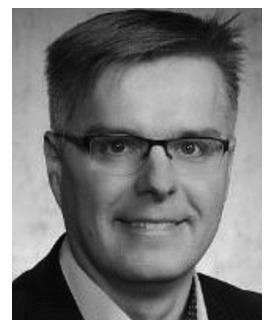

Jussi T. Sopanen (M'14) was born in 1974 in Enonkoski, Finland. He received the M. Sc. degree in mechanical engineering and the D. Sc. (Technology) degree from LUT University, Lappeenranta, Finland, in 1999 and 2004, respectively. He has been a Researcher with the Department of Mechanical Engineering at LUT University during 1999-2006. He has also worked as a Product Development Engineer in electric machine manufacturer, Rotatek Finland Ltd., from 2004 to 2005. During 2006-2012, he worked as Principal Lecturer in mechanical engineering and Research Manager with the Faculty of Technology in Saimaa University of Applied Sciences, Lappeenranta, Finland. He is currently serving as a Professor in Machine Dynamics Lab at LUT University. His research interests include rotordynamics, multi-body dynamics, and mechanical design of electrical machines. 